\title{
Vaccination against fungal diseases: lessons from Candida albicans
}

\author{
Paula Sampaio and Célia Pais
}

\begin{abstract}
(150 to 250 words)
The advances in medicine have achieved great benefits by improving or even eliminating various debilitating diseases or malignancies, expanding life expectancy. However, it has also originated the development of a compromised population susceptible to opportunistic diseases. A global major concern is the emergence and spread of life-threatening invasive infections in immunocompromised patients, in which the opportunistic fungal infections have greatly increased in the last decades. The high mortality rates associated with these infections, which remain as high as $40 \%$, are due to the limited therapeutic options and the emergence of drug resistant fungi, but also due to the lack of efficient early diagnosis. Consequently, these facts led to the opinion that new approaches are needed to improve the outcome of these patients, such as immunopreventive strategies that could even be combined with standard antifungal treatment. In view of the proven effectiveness of various antibacterial and antiviral vaccines in preventing the respective diseases, several works have been developed to induce protective immunity against fungal infections as well. The better understanding of how the immune system works against fungal pathogens has made possible to explore immunomodulatory strategies that can protect both immunocompetent and immunocompromised hosts and generate memory. Recently, two fungal vaccines against Candida have advanced through clinical trials. However, there are still many challenges in the development of an efficient vaccine against invasive fungal infections. We will provide an update on the progress made in immunization against fungal infections, reviewing host-fungi interactions, antigens and adjuvants exploited in vaccine strategies, and discuss concerns that need to be overcome to further advance in the area of fungal vaccines.
\end{abstract}

\section{Candida and its relation with the host}

In healthy individuals, Candida colonization, as part of the normal human microbiota, remains generally benign without any symptoms of disease $[1,2]$. Tolerance of the immune system towards commensal species and maintenance of a homeostatic balance at the mucosal surfaces reflects a series of strategies such as avoidance of immune recognition, active suppression of the host response, and regulation of the immune response by the host, as well as the normal mucosal mi- 
crobiota, confining microbes to this surface [2, 3]. However, when tissue homeostasis is disrupted, Candida might penetrate the forbidden compartments of host tissues and cells, causing pathologies, ranging from recalcitrant skin/mucosal infections to life-threatening systemic infections. The deep-seated or the disseminated infections have increased in frequency particularly in patients with medical conditions and treatments that affect natural barriers of the body or negatively impact the competence of the immune system. Such treatments include the use of broad-spectrum antibiotics, prosthetic devices and grafts, burn injuries or surgery, and medical conditions refer to patients with leukemia, neutropenia or under chemo and immunosuppressive therapies and patients with HIV infection [4]. The current methods to protect susceptible individuals from these opportunistic infections include prophylactic use of antifungal agents or protective environments such as rooms with controlled air flow. Although it is accepted that early, presumptive treatment of patients with invasive candidiasis is a major determinant of survival, such strategies have not been validated by prospective studies [5].

In Intensive Care Units (ICU) Candida spp colonization may occur in up to $80 \%$ of critically ill patients, but curiously, the proportion of invasive candidiasis is less than $10 \%[6,7]$. Therefore, the immune system is important for the maintenance of integrity at the mucosal surface and, even in an immunocompromised state, Candida species remain in the benign commensal state rather than in the invasive pathogenic one. Indeed, some studies suggest that $C$. albicans may evolve to avoid confrontation with the host, favoring commensalism [8, 9]. How the immune system distinguishes these dual behavior, commensal vs pathogenic is a fundamental question in the development of immune preventive strategies against commensal species, and particularly Candida species.

The development of new immunotherapies, including vaccines, is driven by the progress made in the understanding of the molecular pathogenic mechanisms of the infectious agent. Understanding how mucosal homeostasis is established, maintained or disrupted during fungal exposure and/or colonization should help to guide the development of new immunotherapies. Knowledge about the immune response against Candida infections has greatly increased in this last decade, with new data and reviews emerging frequently [10-16]. Thus, a brief review to the immune responses to Candida developed at the mucosa surface will be presented.

\subsection{Within the mucosal layers: interaction of Candida albicans with the epi- thelium}

The mucosal epithelium is a critical barrier and the frontline of sensing microbes. Due to its commensal nature the initial interaction of Candida with the human immune system is frequently via epithelial cells of the mucosa $[17,18]$. There are multiple ways to contain $C$. albicans in the mucosa, such as the microbial flora or the active role of epithelial cells that produce antimicrobial peptides [15]. In addiction, epithelial cells secrete mucins that establish a protective film, forming a dense lattice close to the epithelial surface in which secreted antimicro- 
bial molecules are embedded [19]. Curiously, it has been described that in the gastric mucosa this mucin film is organized into two layers, an inner layer (50 $\mu \mathrm{m}$ thick) that is densely packed, firmly attached to the epithelium and devoid of microbes, in contrast to the outer layer ( $100 \mu \mathrm{m}$ thick) that is movable, has an expanded volume due to proteolytic cleavages of the mucins and is colonized by microbes [20]. Recently, it was described that mucin induces an oval-shaped morphology in C. albicans in which genes related to adhesion, filamentation, and biofilm formation are downregulated [21] and that mucin has an inherent direct candidacidal activity [22]. Mucosal epithelial cells express innate immune receptors or pattern-recognition receptors (PRRs) that recognize microbe-associated molecular patterns (MAMPs), allowing the immune system to detect attachment of microbes and act accordingly. However, the PRR that mediates recognition of fungi in epithelial cells are still largely unknown or undefined. The major known PRRs involved in the recognition of Candida cells were identified in the phagocytic cells and includ the Toll-like receptors (TLRs), C-type lectin receptors (CLRs), NOD-like receptors (NLRs) and RIG-I-like receptors (RLRs) [15]. Among the 13 TLRs discovered, expression of TLR1 through TLR9 has been identified in human intestinal epithelial cells (IECs) [23], but its expression varies in different organisms and different mucosal surfaces (oral, vaginal or gastric) [17]. In addition, TLR2 and TLR4 that are important for host anti-Candida defences $[14,24]$ have demonstrated hyporesponsiveness to their ligands in human IECs [23]. Furthermore, by using a model of oral reconstituted human epithelium (RHE) TLR2, and particularly TLR4, seem to be highly responsive to Candida MAMPs [25]. Expression of CLR in epithelial cells seems to be restricted to dectins. Human IECs express functional Dectin- 1 that recognizes fungal $\beta$-glucans [26], and bronchial epithelial cells also express Dectin-1, which plays an important role in the control of Aspergillus fumigatus infections [27]. As a result of the long co-evolution of fungi, and particularly $C$. albicans with mucosal surfaces having particular environments, the cross-talk between fungi in the different mucosal surfaces has evolved in multiple ways under the double constraints of sensing microbes and adjusting the immune response to the perceived degree of threat while maintaining homeostasis at the mucosa.

Fungal cells differ from animal cells primarily due to the cell wall that surrounds their plasma membrane. The Candida cell wall structure has two distinct layers, an outer layer mainly composed of mannan and mannoproteins $(\mathrm{O}-$ and $\mathrm{N}$ linked glycoproteins), and an inner layer that contains the skeletal polysaccharides chitin, $\beta$-1,3-glucan and $\beta$-1,6-glucan, which confer strength and shape to the yeast cells [28]. These cell wall molecules, which are absent from the human body, are the major source of MAMPs for recognition by host PRRs. However, much of these molecules are expressed in both the commensal state and in the pathogenic state. Recently it was suggested that the pathogenic state may be recognized by "patterns of pathogenesis" or POP rather than only by microbial molecular structures [29]. In this hypothesis, MAMPs are delivered along with additional information that can be used by the host to distinguish pathogenic from non- 
pathogenic state and thereby guide the ensuing innate immune response. During such pathogenic behaviors, MAMPs access forbidden compartments of host tissues and cells, being recognized by PRRs as genuine pathogen-associated molecular patterns (PAMPs) and elicit an appropriate immune response. This POP hypothesis does not define a pathogen, but a pathogenic behavior that can be adopted by any microbe. This allows a particular microbe to be considered a commensal or a pathogen, depending on time and context. Its pathogenicity is determined by its interaction with the host, and can change with host, location in the host, and the immune state of the host [3]. The cross-talk between Candida albicans and the immune system can also be integrated into this hypothesis. It is well established that Candida show different behaviors, the pathogenic (invasive) and the nonpathogenic (commensal) [1, 15, 30], but favoring commensalism [8, 9].

One sign of POP that has been defined is the microbial over-growth in their host [3]. Epithelial cells are able to actively monitor the density of resident microbiota by sampling microbial molecules through PRRs [23]. It is of particular significance that mucosal epithelial cells are able to discriminate between the commensal and the pathogenic behavior of $C$. albicans cells, and there are several ways to control yeast over-growth $[11,31,32]$. The majority of the epithelial TLRs and Dectin-1 signal through MyD88 adaptor, leading to a transcriptional response primarily dependent on the transcription factor NF- $x \mathrm{~B}[15,23]$. In commensalism the presence of $C$. albicans yeast or hyphae triggers the NF- $x \mathrm{~B}$ signaling pathway and an early response of the mitogen-activated protein kinase (MAPK) activation through ERK1/2 and JNK signaling. At the mucosal surfaces this response leads to the secretion of IL-22, by innate and adaptive immune cells, that stimulates the proliferation of epithelial cells and, together with IL-17, the production of antimicrobial peptides, controlling fungal growth and tissue homeostasis $[12,31]$. However, when $C$. albicans outgrows and a high fungal hyphal burden is present the threshold level of the MAPK activation is reached and a second pathway, the MAPK/MKP-1/c-Fos is activated [31]. Activation of this second response results in the production of proinflammatory cytokines, such as interleukin $1 \alpha / \beta$ (IL-1 $\alpha / \beta)$, IL-6, GM-CSF, TNF- $\alpha$ and chemokines, RANTES, IL8 , and CCL20 that induce the recruitment, differentiation, and activation of various immune cells, including phagocytes $[11,31]$. This immune activation results in reduction of fungal burdens back down below the threshold level of activation and thus to the commensal state. One additional way to modulate $C$. albicans population density is through quorum sensing, in which molecules produced during continuous $C$. albicans growth, act as inhibitors of the yeast-to-hypha transition $[1,33]$, and both morphological forms are known to be required for virulence [34]. Curiously, the recently described non-lytic expulsion of internalized hyphal Candida cells by macrophage after phagocytosis benefits not only the immune cells, avoiding their own lysis, but also the fungus as it escapes from intracellular death [35]. This suggests that re-establishment of homeostasis does not implicate the complete elimination of the fungus, but rather reduction of its burden to the commensal state. Thus, at the mucosal surface there is a constant cross-talk between $C$. 
albicans and host mucosal immune system in order to keep the fungal burden below a certain threshold and maintain homeostasis [31].

Another POP behavior is cytosolic invasion, as many pathogens deliver active proteins, or virulence factors to access forbidden compartments of host tissues [3]. When perturbations at the mucosa surface occur, such as the composition of the competitive commensal bacteria that may result from antibiotic use, or when the immune system becomes severely weakened by factors such as HIV infection, chemotherapy, or chirurgic interventions, the proper restriction of $C$. albicans may fail as well as the capacity to control its over-growth, predisposing individuals to Candida invasion. If containment at the mucosa is breached, the yeast proliferates and penetrates forbidden compartments of the host tissues. Candida albicans has the ability to invade host epithelial cells by two routes: via active penetration and via induced endocytosis $[36,37]$. The active penetration is exclusively a fungal attribute and includes the physical pressure applied by the advancing hyphal growth in combination with the secretion of extracellular hydrolases like proteases, phospholipases and lipases $[15,38]$. The induced endocytosis is the passive uptake of C. albicans cells through yeast surface proteins Als3p and Hgclp by the Ecadherin receptor in host epithelial cells [38].

As stated above, the epithelial cells actively monitor resident microbes by sampling microbial molecules and channels that transport microbial cell wall components into epithelial cells [23]. To aid in the consequent epithelial response, several PRRs are intracellular, cytoplasmic, and are involved in pathogen recognition, antigen processing and presentation, production of antimicrobial $\beta$-defensins, and activation of the inflammasomes. The inflammasome is a multiprotein complex that triggers the post-translational activation of caspase-1 that in turn cleaves and activates the secretion of a variety of substrates, including pro-interleukin-1 $\beta$ and pro-interleukin-18, leading to an inflammatory response [15]. These PRRs receptors are not exposed to pathogens unless they get into the cells, when invading mucosa, like TLR3 and TLR9 that recognize fungi nucleic acids, or NOD2 that recognize fungal chitin $[39,40]$. It has also been reported that this activation may induce the maintenance of a minimal level of "physiological inflammation" by activating NOD proteins in the cytoplasm of gut epithelial and immune cells. Curiously, recognition of fungal chitin by NOD2, together with TLR9 and the mannose receptor, are essential for the selective secretion of the anti-inflammatory cytokine IL-10 by phagocytic cells [41]. This exerts a collaborative control in which TLR signaling is required for the transcription of pro-IL-1 $\beta$ and pro-IL-18, and the post-translational inflammasome response is then required for subsequent cleavage and secretion of the active cytokine. This dual control may be an important regulatory mechanism to limit inappropriate or unnecessary production of cytokines that trigger potent responses that may be potentially damaging to the host, particularly concerning commensal organisms. Thus, the immune system can initiate responses based not only on whether PAMPs are present, but also on where those PAMPs are presented, allowing the epithelium to adjust the response 
to the perceived degree of threat and return to the homeostatic state once the pathogen has been controlled.

\subsection{Beyond the mucosal layers: overview of the immune response against Candida infections}

At the mucosal epithelium, Candida may assume a fully pathogenic behavior, invading deeper tissues, gaining assess to the blood stream and causing invasive infections $[11,12]$. One of the causes of this drastic rupture of homeostasis is a too weak immune system. The life-threatening opportunistic Candida infections occur particularly in immunocompromised patients in which the immune forces that normally contain $C$. albicans and establish equilibrium are at risk of being overwhelmed.

The host immune system responds with the innate immune attack as the immediately acting primary line of defense against fungi penetration. Recognition of fungal-associated PAMPs will lead to the initiation of a host defense that is mandatory to limit fungal burden in order to clear the invading fungus from nonauthorized sites $[11,15]$. The cell populations that orchestrate this initial response include the phagocytic cells: neutrophils, macrophages and dendritic cells (DCs) $[10,13,15]$.

Macrophages are considered the first line of defense since they are distributed in various tissues as resident cells. Macrophages exhibit a considerable phenotypic diversity and functional plasticity that confer them the ability to efficiently respond to stimuli. The fungal cell wall is a dynamic structure that is continuously changing throughout the fungus cell cycle and during morphological transition. During tissue invasion macrophages readily ingest the round yeast form of $C$. albicans as well as relatively short filaments. The development of hyphae triggers the recognition of cell wall $\beta$-glucans via the Dectin-1/inflammasome pathway. Engagement of Dectin-1 induces not only direct macrophage activation and internalization of the fungus but it also has a synergistic effect on TLR2 and TLR4 responses, via NF- $\varkappa \mathrm{B}$ activation, leading to IL- $1 \beta$ production and $\mathrm{T}$ helper cell 17 (Th17 cell) activation [32, 42, 43]. However, upon hyphal growth $C$. albicans is able to shield $\beta$-glucans with surface mannans as a mechanism to evade cellular phagocytosis [18]. Thus, other PAMPs, such as mannans and mannoproteins, are also recognized by several CLRs, including Mannose Receptor, Dectin2 , dendritic cell DC-SIGN, and MINCLE [14, 44]. Dectin-2, mainly expressed on dendritic cells, macrophages and neutrophils recognizes Candida $\alpha$-mannan and, together with Dectin-3 has been reported to form heterodimers, mediating Candida uptake by the phagocyte and leading to pro-inflammatory responses during C. albicans infection, also via NF-xB activation [10,45].

In consequence of the inflammatory response neutrophils migrate to the site of infection and after recognition of Candida cells, they can directly kill the yeast cells, inhibit their growth and the yeast-to-hyphal transition, limiting in this way the progression of fungus [46]. The importance of these phagocytes is highlighted by the observation that the depletion of mononuclear phagocytes results in 
accelerated fungal proliferation in tissues and increased mortality [47], and that neutropenic patients or patients with impaired neutrophil function are more susceptible to systemic fungal infections [46]. Phagocytic clearance of $C$. albicans involves the accumulation of phagocytes at the site where the yeast cells are located, and engulfment and killing of fungal cells after the recognition of cell-surface fungal PAMPs [48]. These innate immune cells damage or kill C. albicans cells by using a combination of oxidative and non-oxidative mechanisms that includes the production of antimicrobial peptides and degradative enzymes, and the generation of antimicrobial reactive oxygen species (ROS)/nitric oxide synthase (iNOS) $[49,50]$. Human neutrophils and macrophages express constitutively chitotriosidase (CHIT-1), an enzyme that promotes digestion of fungal cell wall chitin, and whose expression increases significantly during the end/late-phase of infection [41]. Curiously, low concentrations of small chitin particles have been shown to induce IL-10 secretion, via NOD2 and TLR9 signalling [41]. This mechanism has been proposed to contribute to the resolution of the infection when the pathogen has been defeated by the immune system, promoting the attenuation of inflammatory-mediated diseases and consequent immune homeostasis.

The innate immune system not only specifically recognizes diverse microorganisms, but also initiates and modulates the subsequent adaptive responses that are delivered by $\mathrm{T}$ cells and $\mathrm{B}$ cells through their interactions with antigenpresenting cells. Phagocytic DCs play an essential role in the activation of adaptive immune responses, being one of the most efficient antigen presenting cells (APCs). DCs are important for processing and presenting fungal antigens but, due to the dual nature of $C$. albicans, their reaction to this fungus must be flexible in order to shape T-cell responses accordingly. Th1 responses involving proinflammatory cytokines, such as IFN- $\gamma$ and TNF- $\alpha$, were considered protective against fungal infections whereas Th2 responses, with the secretion of IL-4 and IL-10, were believed to enhance host susceptibility [16]. However, with the identification of Th17 response, involving proinflammatory cytokines IL-17 and IL-22, this is now considered a major pathway for protection against fungal infections [12]. These cytokines induce neutrophil recruitment and activation, as well as the activation of epithelial cells and release of antifungal $\beta$-defensins, being important at the mucosal surface. The importance of Th17 in anti-Candida response was confirmed by the identification of functionally specific Th17 clones that respond to $C$. albicans by releasing IL-17 and IFN- $\gamma$, but not IL-10 [51]. Indeed, patients with defects in Th17 immunity have been directly linked to increased susceptibility to chronic mucocutaneous candidiasis (CMC) [52]. After recognition of Candida, the plasticity of DCs is reflected into distinct signaling pathways that are translated into different in vivo adaptive immune responses [53]. Inflammatory DCs initiate antifungal Th17/Th2 cell responses to yeast through signaling pathways that involve adaptor MyD88, whereas tolerogenic DCs activate Th1/Treg (T regulatory) cell responses to hypha through TRIF adaptor $[16,53]$.

The balance between proinflammatory and anti-inflammatory signals is essential for successful host/pathogen interaction in fungal infections in which 
Treg cells play a critical role [53]. Treg cell, essentially via production of IL-10, are able to inhibit components of innate and adaptive immunity in order to regulate the host's immune defense to an adequate level; a protective response without total elimination of the fungus and preventing an exacerbated level of tissue damage $[16,53]$. However, overly potent Treg suppression can inhibit protective immunity, favoring the pathogen. Nevertheless, since Treg cells control the quality and magnitude of innate and adaptive effector responses the outcomes may range from protective tolerance to overt immunosuppression, determining the balance between commensalism and pathogenicity. At the mucosa, a local antiinflammatory/tolerogenic response through secretion of IL-10 and TGF- $\beta$, would allow for the fungus persistence, whereas beyond the mucosa, an appropriate inflammatory immune response, is necessary to eliminate the fungus and return to a homeostasis state.

The role of Th17 responses in systemic fungal infection is somehow controversial since, on one hand it is described that IL-17RA-/- and IL-17A-/- mice have elevated susceptibility to disseminated candidiasis [54, 55] and IL-17A mediates vaccine induced protection in mice [56]. On the other hand, humans with mutations in the IL-17 pathway typically do not develop disseminated disease [55] and candidemic patients showed significantly higher levels of IL-17A than patients without invasive candidiasis [57]. The idea that elevated Th17 and Treg responses are harmful in disseminated candidiasis seemingly contrasts with the apparent protective role of IL-17 in mice. In disseminated candidiasis it has been reported that $C$. albicans drives expansion of a complex Treg cell population that by depletion of IL-2 promotes the development of Th17 responses that are associated with immune pathology and with $C$. albicans survival and dissemination [58] [55].

Th17 and Treg subsets are reciprocally regulated during naïve $\mathrm{T}$ cell differentiation. However, it is clear that IL-17/Th17 and Treg cells have a complex relationship and that the outcome of the response to Candida depends on the site of infection in which the corresponding microenvironment is able to shape these responses. At the intestinal mucosa, high levels of transforming growth factor beta (TGF- $\beta$ ) and retinoic acid have been found that together with short chain fatty acids (SCFAs) from commensal microbes fermentation support tolerogenic Tregs, favoring $C$. albicans commensalism. In contrast, internal organs are shielded from the external environment, and thus inflammation induced during disseminated $C$. albicans infection is more likely to go unchecked, resulting in collateral tissue damage by Th17 responses [55, 58]. Together, these data underline the importance of the balance between proinflammatory and anti-inflammatory responses for both susceptibility to acquire invasive candidiasis and ability to clear the infection, once it has disseminated.

In addition to the cellular responses adaptive B-cell, by inducing antibody mediated memory responses, may neutralize adherence and spread on and/or through epithelial and endothelial tissues. The role of antibodies in the defense 
against candidiasis has been controversial due to the believe that immunocompromised patients, the main targets of opportunistic fungal infections, were unable to develop adequate adaptive B-cell response. In addition, due to the fact that Candida are commensal organisms, natural anti-Candida antibodies may be present in the serum of normal individuals but no correlation with these antibodies and protection to a subsequent infection has been observed.

There is now substantial evidence for the important role of antibodymediated immunity in clearance of infectious agents, including host resistance to systemic candidiasis. Regarding the class of immunoglobulins, it has been proposed that IgM titers against intracellular proteins that are not localized to the cell wall would be higher in patients with systemic candidiasis than in controls, which suggests that these antigens might be less visible to the humoral immune system during commensal growth [59]. However, Regarding the subclass, a recent study evaluated how the IgG subclass of an anti-Candida antibody influences its biological functions, using the mouse model [60]. Four IgG subclass variants of the human antimannan antibody, Mg1, M1g2, M1g3, and M1g4, equivalent to IgG1, $\mathrm{IgG} 2, \mathrm{IgG} 3$, and $\mathrm{IgG} 4$, were constructed and evaluated. They found that the four IgG subclasses differ in protection against hematogenously disseminated candidiasis in mice, with IgG1, IgG3, and IgG4 being more effective than IgG2. This difference has been attributed to the lower capacity of IgG2 to mediate Fc $\gamma \mathrm{R}$ dependent phagocytic clearance. All variants promoted deposition of both murine and human complement $\mathrm{C} 3$ onto the yeast cell surface however IgG4 was less potent in activating the complement system. The fact that IgG4 was less potent in activating the complement system but induced similar levels of protection than $\mathrm{IgG} 1, \mathrm{IgG} 3$, and was even more protective than $\mathrm{IgG} 2$, suggests that the role of the complement system in mediating protection may not be that determinant. Indeed, in a murine model of cryptococcosis it was found that complement-nonactivating human IgG2- or IgG4-mouse chimeric antibodies are protective, while complement-activating human IgG1- or IgG3-mouse chimeric antibodies are nonprotective [61].

The study of the immunoglobulins present in the serum of human subjects without signs of infection in comparison with patients with proven systemic candidiasis has also contributed to the identification of proteins that could be specifically recognized in each situation. The antibodies identified specifically in subjects without infection were considered as natural anti-Candida antibodies and are believed to arise from the commensal relation with the host. Some of the natural anti-Candida antibodies identified Candida, Eno1p, Pgk1p, Adh1p, and Pdc11p, are abundant metabolic enzymes present in the yeast cell wall [62]. However, only antibodies against Pgk1p and Adh1p seem to differentiate patients with systemic candidiasis from non-infected subjects. Antibodies against glyceraldehyde-3phosphate dehydrogenase (Gaplp), phosphoglycerate kinase (Pgk1p), phosphoglycerate mutase (Gpm1p), enolase (Eno1p), pyruvate kinase (Cdc19p), aconitase (Aco1p), methionine synthase (Met6p), hyphal wall protein (Hwp1), mannan, among others have been identified specifically in patients with systemic candidia- 
sis. However, conditions that make the molecular targets available to the immune system to induce antibodies differ according to many variables, including the morphology changes of the pathogens, the competence of the immune system at the entry port, the composition of the commensals and their similarity of epitopes, and the history of antimicrobial treatments that have different modes of action, among others. All these mechanisms contribute to expose antigenic molecules to the immune system and some treatments will increase/alter exposure of certain antigenic molecules. This may be one of the reasons why it has been so difficult to identify antibodies/antigenic molecules that would be useful for immunoglobulinbased prophylactics and therapeutics. Nevertheless, Mycograb (NeuTec Pharma) a human recombinant antibody anti-Hsp90p was found to be therapeutically effective in combination with amphotericin B in patients with systemic candidiasis in a randomized, blinded, multicenter trial [63].

These studies evidence that the role of antibody-mediated resistance to fungal infections is important and may be exploited as new therapeutic approaches however, the isotypes that could be more effective in a response that involves antibodies must be carefully chosen to mimicking the host's natural protective antibody and balance between its disease-causing effects, as exacerbated complement activation, and its effects on clearance of microbes.

Intense investigation of immunological mechanisms involved in fungal infections means that we are closer to understanding the processes involved in the identification and clearance of pathogens from the human body and in establishing immune memory to respond to future exposure. The ideal vaccine would initiate an innate immune response capable of directing the adaptive immune response toward efficient inactivation and removal of the specific pathogen from nonallowed sites, followed by the development of adequate immune memory. In the next section, an up-date of the studies toward the development of vaccination strategies will be presented.

\section{Induction of immunity in experimental models}

\subsection{Concerns about the development of preventive strategies}

The increasing incidence and severity of invasive fungal infections coupled with diagnostic difficulties and high cost of treatments prompted the investigation on vaccination [64]. However, for years the development of fungal vaccines has lagged behind that of vaccines against bacteria and viruses because of the idea that most patients who develop life-threatening fungal infections have profound defects in their immune systems and are unable to respond to vaccination. Indeed invasive Candida infections often takes place in hospital settings, however it is has been estimated that only $10-20 \%$ of patients who develop bloodstream infection from Candida are seriously immunocompromised. The large majority develop the infection after patient's normal bacterial flora is disrupted by antibiotics, or when their skin or gut mucosa are breached by burns, central ve- 
nous catheters or surgery $[4,5,65]$. Furthermore, several studies confirmed the immunogenicity and efficacy of vaccines in seriously immunocompromised patients, such as those with active leukemia, HIV infections, or cancer patients [6668]. Thus, immunocompromised patients are able to respond to vaccination and vaccination has been recommended to patients with HIV and cancer despite their weakened immune systems [69-71]. It has been assumed that acutely ill hospitalized patients whose primary host defense defect is anatomical will also respond to vaccination [65]. In the case of Candida systemic infections the various medical procedures that are identified as risk factors, such as exposure to broad spectrum antibiotics, central venous catheters or major surgery are perfectly recognized, opening a window of opportunity to vaccinate at-risk patients even before the onset of infection [72-74]. In addition, prevention of infectious disease in the elderly has become a priority in the 21 st century as the proportion of older individuals increases globally. Fungal infection, due to Candida, Aspergillus and Cryptococcus are known to be more frequent in the extreme of the ages, children and elder patients.

Consistent with Benjamin Franklin's dictum 'an ounce of prevention is worth a pound of cure', preventive therapies against invasive candidiasis may reduce the severity of these infections. A vaccine that could prevent or improve the outcome of fungal infections would not only benefit the at-risk patients but also be of major benefit for national healthcare systems by reducing hospital costs.

\subsection{Evidences from systemic candidiasis in the human host}

Several studies were undertaken to identify Candida albicans immunogenic proteins specifically recognized by antibodies produced during the natural course of systemic Candida infection and that could be exploited for vaccine development or even as useful biomarkers for diagnosis. One of the earliest studies evaluated, by immunoblotting, 201 sequential serum samples from 45 patients with systemic candidiasis in which 13 were involved in an outbreak at London Hospital [75]. This study showed a high heterogeneity in antibody response but was able to identify an antibody against a $47 \mathrm{kD}$ antigen present in all patients that recovered from the systemic infection. Latter, the $47 \mathrm{kD}$ antigen was identified as a breakdown product of heat-shock protein (Hsp) 90 from C. albicans. The presence of antibodies against a $47 \mathrm{kD}$ antigen in serum of other patients with systemic candidemia with a favorable outcome has also been identified by other independent studies. Serum from these patients passively transferred to mice infected with a lethal dose of C. albicans was able to induce protection, and a monoclonal antibody raised against a synthesized conserved epitope (LKVIRK) of Hsp90p reduced mice mortality when given $24 \mathrm{~h}$ before an i.v. challenge [76]. This antibody seems to protect through neutralization of the protein-binding activity of Candida circulating Hsp90p or by inhibition of Candida growth [77]. However, an epitope mapping analysis indicated that antibodies reacting to epitopes of this protein showed $100 \%$ direct homology with comparable sites on human Hsp90p, raising the con- 
cern of development of autoimmune reactions. These studies showed that patients who recover from systemic candidiasis may produce autoantibodies against selfepitopes.

More recently another study used a highly sensitive proteomic approach to identify potential candidate markers for diagnosis and follow-up of systemic candidiasis [62]. This study identified immunoreactive protein species that were present in patients with proven candidemia and also in patients with no apparent Candida infection. In patients with invasive candidiasis the authors were able to unambiguously identify 42 different $C$. albicans protein targets of the human immune response. The abundance of housekeeping enzymes, most of them $(65 \%)$ metabolic enzymes was discussed, as among other possibilities, being part of an universal signal for infection. Since these are ubiquitous and highly conserved enzymes their epitopes can be shared by several infectious agents and allow the development of a natural resistance to infection. The identifications of these proteins by the immune system may be considered as part of the "patterns of pathogenesis" response. Among the metabolic enzymes, that are also present on the $C$. albicans cell surface, antibodies against Glyceraldehyde-3-phosphate dehydrogenase (Gaplp), Phosphoglycerate kinase (Pgk1p), Phosphoglycerate mutase (Gpmlp), Enolase (Enolp), Pyruvate kinase (Cdc19p), Aconitase (Acolp), Methionine synthase (Met6p) had already been identified in human serum previously. Several other proteins, including Hxk2p (hexokinase) and Pgilp and 6phosphofructokinase were identified as antigens of the immune system for the first time in this study. Curiously, antibodies against C. albicans proteins Eno1p, Fba1p, Tk11p, Ino1p, Met6p, Sah1p, Ilv5p, Ade17p, Qcr2p increased in response to antifungal treatment, indicating that their exposure to the immune system increased with the antifungal treatment. From this survey $14 \%$ of the identified targets were chaperons and heat shock proteins, including Ssa1p and Ssb1p identified in previous studies as antigens, and Ssc1p and Sse1p newly identified as antigens in this study. Curiously, the 47-kDa fragments of Hsp90p identified previously [75] was not identified in this analysis, only the $82-\mathrm{kDa}$ fragment of the same protein. The C. albicans elongation factor 1 (Eft1p) and Eft2p, known to be located in the cell wall and proposed as potential antigens for vaccine development were also found in this study.

The protein targets identified in the serum of subjects with no infection were considered as targets of natural anti-Candida antibodies. These natural antibodies identified Candida Eno1p, Pgk1p, Adh1p, and Pdc11p, which corresponded to abundant glycolytic and fermentative enzymes. The natural antibodies were considered to be elicited during commensal colonization by C. albicans or against other homologue protein from different commensals or infectious agents that cross-react with C. albicans proteins. This study also identified targets that could be correlated with recovery from systemic candidiasis in humans, like high serum levels of anti-Enolp antibodies, the appearance or maintenance of anti-Hsp90p, anti-Tpilp, or the disappearance of serum anti-Met6p or anti-Pgk1p antibody levels. 
Another study measured IgG and IgM titers in the serum of human systemic Candida infections against 15 specific recombinant $C$. albicans antigens [59]. It is documented that patients with systemic candidiasis already exhibit significantly high IgG titers against a wide range of antigens (ENO1, HWP1, mannan, and CGTA) before or at the time that the diagnosis is confirmed by a positive blood culture. This study suggests that invasive candidemia may be preceded by lowlevel systemic exposure to Candida spp., possibly due to "leakage" from mucosal sites of colonization.

\subsection{Exploited protection strategies against candidiasis}

\subsubsection{Whole cells vaccines}

The need for therapeutic or preventive use of a vaccine in candidiasis, particularly in certain groups of at risk patients is reflected in the huge number of experimental investigations designed to attempt induction of protection. The early studies trace back to the decade of 50's.

One of the early documented studies repeatedly immunized rabbits intravenously (i.v.) with heat-killed $C$. albicans prior to infection and then assessed survival, histopathology of infected organs and passive immunization. The authors concluded that this immunization did not result in protection and that the antiCandida antibodies induced by vaccination did not protect the animals [78]. Latter, by using the same model but immunizing with heat-killed and heat-weakened Candida, protection was only observed in rabbits immunized with viable Candida, and histological analysis indicated that cellular immunity was important [79]. These observations point to the active action of the pathogen in the immunization process. In the murine model low virulent Candida strains, modified in the filamentation capacity and stress response, where shown to provide protection against a subsequent intravenous systemic Candida infection [80-82]. The efficacy of these strategies reached $100 \%$ for all strains at least in one evaluated condition. Other study, using the mouse model, also observed the importance of fungal pathogenicity on priming of mice with Candida [83]. Naive BALB/c mice were intraperitoneally (i.p) primed with a low inoculum of Candida yeast cells that were heatkilled, deliberately attenuated or in its pathogenic native form. One month later mice were infected with the pathogenic strains and only the mice primed with the pathogenic native form were able to clear the infection. Live-attenuated pathogens or in its pathogenic native form have been shown to be especially immunostimulatory when used as vaccines. This may be explained by the hypothesis that the immune system also responds to POP and suggest why killed strains failed to elicit protection [29]. However, due to the complexity of antigens and the inexistence of generalizable procedure for how best to attenuate pathogens for their use as vaccines, attenuated strains challenge safety requirements. However, changing to a subcutaneous (s.c.) immunization with a Candida sonicate, Mourad and Friendman (1968) were able to protect $50 \%$ of mice, obtaining the best result in compar- 
ison with viable or killed $C$. albicans cells. In addition, they observed that transferring passively anti-Candida serum to naïve mice conferred them protection against a subsequent systemic candidiasis [84]. After these results, subcellular antigens have been further exploited as candidates for augmenting host defense and vaccine development against candidiasis.

\subsubsection{Ribosomal vaccines}

Ribosomal subcellular vaccines derived from both procaryotic and eucaryotic pathogens were widely exploited as protectors and afforded high levels of protection at small dose levels, being the duration of the immunity they provided greater than the ones obtained with previous antigens used. Thus, ribosomal fractions were also used to try to induce protection against candidemia. Studies documented that it was possible to induce protection by immunization with $C$. albicans ribosomes, not only against heterologous $C$. albicans strains, with $30-78 \%$ of survival, but also cross protection against $C$. tropicalis isolates, with 57 to $64 \%$ of survival rate from a systemically administered lethal infection [85]. Ribosomal vaccine was also exploited in experimentally induced cell-mediated immunocompromised mice, resulting in a survival rate that reached $76 \%$ [86]. This vaccine was also exploited in tumor-bearing mice and although the survival rate did not change, it was able to expand the median survival time [87]. These results showed that vaccination of immunocompromised and tumor-bearing hosts with Candida ribosome could provide enhanced resistance to disseminated candidiasis. These works encouraged the continuing studies in immunization against candidiasis and revealed the immunogenicity of subcellular components and the role of antibodies in the protection. A variety of cell constituents, routes of administration and protocols have been tested and despite the degree of variability in the results observed, induction of protection was considered possible. However due to the complexity of the antigen, that would probably include mRNA, rRNA and ribosomal proteins, newly synthesized polypeptides along with other cell components from the extraction procedure, the identification of the moiety to fully describe the protective immunity mechanism was difficult and other more defined antigens have been exploited.

\subsubsection{Cell wall component vaccines}

Fungal cell wall constituents are excellent candidates for vaccine development since the cell wall is a unique microbial feature with an important role in antigen presentation and immunomodulation [28]. Candida albicans cell wall is composed mainly of carbohydrates (80-90\%), such as mannans, $\beta$-glucans and chitin, while proteins and lipids are a minority. $\beta$-glucans and chitin are located at the innermost layer of the cell wall, providing structural rigidity and strength, while mannans are abundant in the outer layer. Purified polysaccharide vaccines are poorly immunogenic and largely ineffective, due to the inability of the polysaccharide to induce T-cell responses. Thus, in glycan-based vaccines it is common to conjugate the polysaccharide to a carrier molecule such as the tetanus or diphtheria bacterial 
toxoid [88], which are accepted for human use, or to peptides derived from the infectious agent of interest [89]. Nevertheless, glycopeptides may still be poorly immunogenic and adjuvant has been also included, like the complete Freund's adjuvant [89]. Previous studies have shown that the major cell wall components that elicit a host immune system response are proteins and mannoproteins, in which both the carbohydrate and protein moieties are important $[89,90]$. Candida surface mannans were used either alone, or encapsulated [91] or even conjugated with protein to induce protection against experimental disseminated candidiasis [92]. The formulations of mannans conjugated with BSA showed high levels of protection with $100 \%$ survival in a disseminated murine model. This study also suggested that di- and trisaccharides of mannans were more efficient than tetrapenta- or hexasaccharides in inducing protective antibodies. In order to avoid the controversy of results that might be influenced by the distinct methods of mannans extraction the new approaches developed fully synthetic mannans conjugated vaccines. In this view a synthetic trisaccharide mannan-tetanus toxoid conjugated vaccine was used in experimental immunization protocol that was designed to simulate the clinical situation in which vaccination can be performed before a patient becomes immunocompromised by treatment with immunosuppressive drugs to induce leukopenia [93]. Although no survival rate was quantified in this study, this conjugate vaccine achieved a statistically significant reduction of fungal burden in kidney and liver of immunized rabbits, when compared to controls and this was attributed to the high titer of specific IgG antibody capable of opsonizing the surface of $C$. albicans cells. This model indicated that even in leukocytopenic animals a previous vaccination is able to induce resistance against disseminated candidiasis.

Xin and collaborators [89] selected $C$. albicans cell surface proteins previously identified as inducers of antibodies during human candidiasis [62] to conjugate with $\beta$-mannan. The selected proteins were fructose-bisphosphate aldolase (Fba), methyltetrahydropteroyltriglutamate (Met6), hyphal wall protein-1 (Hwp1), glyceraldehyde-3-phosphate dehydrogenase (Gap1) and phosphoglycerate kinase (Pgk1). By using an antigen-pulsed DC-based vaccine strategy, immunization with $\beta$-(Man)3-Hwp1, $\beta$-(Man)3-Fba, or $\beta$-(Man)3-Met6 conjugates showed 80$100 \%$ survival rate, while with $\beta$-(Man)3-Enol or $\beta$-(Man)3-Gap1 the survival rate was 40 or $80 \%$, respectively. Curiously, in this study $\beta$-(Man)3-Pgk1 slightly enhanced disease. The $\beta$-(Man)3-Fba conjugate vaccine, that induced the highest protection, showed similar results against disseminated candidiasis in two different mouse strains, BALB/c and C57BL/6 mice. However, the immunization protocol utilizing dendritic cells is cost prohibitive. Thus, $\beta$-(Man)3-Fba has been exploited in a subcutaneous immunization protocol using different adjuvants. After several approaches using of $\beta$-(Man)3-Fba with alum or MPL (Lipid A, monophosphoryl) with no significant protection, the use of tetanus toxoid was able to restore protection previous observed and even protect $100 \%$ outbred mice [94].

The protective responses against Candida mannan components appeared to be mainly by induction of protective antibodies specific against the fungal peptide 
epitopes and the conjugated polysaccharides, which enabled the production of anti-polysaccharide IgG antibodies in vivo. The switch from $\operatorname{IgM}$ to $\operatorname{IgG}$, the activation of the complement system and the rapid deposition of high amounts of factor $\mathrm{C} 3 \mathrm{~b}$ onto the yeast cell wall are mechanisms that contribute to inhibit Candida growth in vitro $[89,94,95]$. These results substantiate the role of antibody in protection against systemic candidiasis in the animal model, evidenced by high survival rates and reduced organ fungal burden.

Although mannoproteins are the major components of the cell wall in eliciting immune responses, $\beta$-glucans were also exploited. However, due to their poor immunogenicity $\beta$-glucans must be also conjugated with other molecules. The brown alga Laminaria digitata $\beta$-1,3-glucans, the laminarin, has been exploited as immunizing antigen conjugated with the diphtheria toxoid and results showed it was protective against both mucosal and systemic candidiasis in mice, with a protective rate around $70 \%$ [88]. Remarkably, this laminarin vaccine strategy conferred cross-protection to mice lethally challenged with the major fungal pathogens C. albicans, Aspergillus fumigatus and Cryptococcus neoformans [96]. The bacterial Calreticulin (Crt) protein is functionally similar to heat shock proteins and one of the main characteristics of this HSP family is that they possess a potent immunostimulatory activity. This characteristic was exploited as an effective carrier or adjuvant for $\beta$-glucans in assisting the production of glycotope-specific IgG antibodies in healthy as well as in immunocompromised mice. Laminarin conjugated with a peptide from Calreticulin (LAM-CRT) induced proliferation of B lymphocytes and production of specific laminarin and $\beta$-1,3-glucans IgG1 antibodies, even in athymic (T-cell-deficient) nude mice [97]. Vaccination with LAMCRT protected mice against systemic $C$. albicans challenge with a survival rate around $60 \%$.

\subsubsection{Vaccines for neutralizing virulence factors}

The previous approaches of vaccination were mainly based on the stimulation of the immune system with whole microbes or with constitutive microbial epitopes present at the surface of the infectious organism. However, the most effective human vaccines, which prevent tetanus and diphtheria, are directed against the bacterial toxins rather than against the structural epitopes. Thus, another approach in the development of immunoprotective therapies against fungal infections is the development of strategies that focuse on the neutralization of virulence factors, either present in the cell wall or secreted that should be produced by microorganisms in their pathogenic form [83]. Virulence factors include proteins involved in the adhesion to and invasion of host tissues, secreted lytic enzymes, the morphogenetic yeast-to-hyphae switching, the maintenance of cellular integrity or the avoidance of the host immune response.

In this view, vaccines based on the adhesins Als1p and Als3p were exploited. Als1p allows binding of C. albicans to human vascular endothelial cells and Als $3 p$ is a hypha-specific surface protein that also mediates attachment to epithelial and endothelial cells. In addition, Als $3 p$ is one of the adhesion molecules that is re- 
sponsible for the binding to host cell receptors, such as E-cadherin, and induce passive endocytose of $C$. albicans by host epithelial cells [38]. Intraperitoneal immunization of $\mathrm{BALB} / \mathrm{c}$ mice with a recombinant $\mathrm{N}$-terminal peptide of Als $1 \mathrm{p}$ $(\mathrm{rAls} 1 \mathrm{p}-\mathrm{N})$ induced modest protection against invasive candidiasis, protecting $25 \%$ of the infected mice [98]. However, when a subcutaneous route of infection was tested, the protection rate was much higher, around 50 to $57 \%$ of immunocompetent mice survive the lethal infection [99]. This vaccination regime was also applied to neutropenic mice that were challenged with a 10 time lower inoculum, with and overall survival of $88 \%$ versus $38 \%$ of the controls, as well as in a mucosal oropharyngeal candidiasis model, with results showing a clear reduction on invasive tongue lesions. The recombinant $\mathrm{N}$-terminal peptide of Als3p (rAls3p-N) was also exploited and compared with rAls1p-N [100]. Results indicated that rAls $3 p-N$ is as effective as rAls $1 \mathrm{p}-\mathrm{N}$ against disseminated candidiasis, with similar survival rates, around 40 to $50 \%$, but was more effective than rAls $1 \mathrm{p}-\mathrm{N}$ against oropharyngeal or vaginal candidiasis. This high effectiveness in mucosal models may be due to the fact that Als3p mediated superior adhesion to epithelial cells than Als1p.

These data showed promising results and rAls $3 \mathrm{p}-\mathrm{N}$ stand out as a candidate for further development of an anti-candidal vaccine. However, these vaccines used Freund's adjuvant to enhance immunogenicity, which is not allowed for human use. In order to switch to an adjuvant approved for human use, these vaccines were tested with alum (Alhydrogel) but the efficacy was lower than with Freund's adjuvant even using a higher dose of the recombinant proteins. The survival rate using Freund's adjuvant ranged from $100 \%$ to $50 \%$ using $20 \mu \mathrm{g}$ of rAls $1 \mathrm{p}-\mathrm{N}$ while with Alhydrogel diluted in phosphate-buffered saline (PBS) was of only $10 \%$ using $100 \mu \mathrm{g}$ of rAls $1 \mathrm{p}-\mathrm{N}$ [101]. Regarding rAls $3 p-\mathrm{N}$, the protection of around 40 to $50 \%$ with Freund's adjuvant shifted to around $35 \%$ with $300 \mu \mathrm{g}$ of antigen with Alhydrogel [102]. Interestingly, Als3p is structurally similar to the Staphylococcus aureus surface clumping factor (ClfA) that is also involved in adhesion, so these authors also tested for a cross-reactivity of rAls $3 \mathrm{p}-\mathrm{N}$ vaccine against $S$. aureus infection in mice. Results showed that vaccination with rAls $3 p-N$ in Alhydrogel improved the survival of mice subsequently infected with multiple clinical isolates of S. aureus (40\% to $70 \%$ of survival rate) [103]. Although rAls $1 \mathrm{p}-\mathrm{N}$ and rAls3p-N vaccines induced broader antibody-based responses, antibody titers did not significantly correlate with survival in both recombinant peptides. Thus, the protective mechanism of these recombinant peptides was not correlated with protective antibodies but was associated to enhancing cell-mediated immunity. In concordance with these observations B-cell-deficient mice showed the same pattern of resistance while in T-cell-deficient (nude) the protection against systemic candidiasis previously observed was abolished.

More recently, the efficacy of rAls $3 p-N$ vaccine with alum as adjuvant in protecting against vulvovaginal candidiasis was evaluated in a murine VVC model [56]. In this model, rAls $3 \mathrm{p}-\mathrm{N}$ induced a strong immune humoral response with high anti-rAls3p-N serum IgG and vaginal IgA titers. In addition, ex vivo killing 
of opsonized $C$. albicans by neutrophils was correlated with significant decrease in vaginal fungal burden both in inbred and outbred mice infected with different clinical C. albicans isolates. As in previous studies, the role of $\mathrm{B}$ and $\mathrm{T}$ cells in vaccine-mediated protection was evaluated in B-cell-deficient and nude mice, respectively, showing that contrary to the systemic model, rAls3p-N efficacy in the VVC model requires both $\mathrm{B}$ and $\mathrm{T}$ cells.

Other virulence factors that were thought to be neutralized were the Secreted Aspartyl Proteinases (SAPs), an enzyme family with 10 members, from Sap 1p to Sap10p. While Sap1p to Sap8p are secreted and released to the surrounding medium, Sap9p and Sap10p remain bound to the cell-surface [2]. These ezymes are involved in the degradation of mucous substrates and defensive factors, promoting Candida damage to epithelial and endothelial cells and helping the fungal cells to elude the host immune response. One of the early studies presented results of immunization against a secreted unidentified $43000-\mathrm{MW}$ protein, named $\mathrm{p} 43$, that resulted in the complete neutralization of its immunomodulatory effects, and was very efficient in protecting mice, i. e. no CFUs were observed in the kidney of vaccinated mice in comparison with the controls [104]. The mechanism of protection involved the production of protective specific antibodies against p43 evaluated by passive administration. In latter studies the same authors used $C$. albicans Sap2p, a $41000-M W$ secreted protein that they believed to mediate some of the effects observed with $\mathrm{p} 43$. However, they concluded that although an immunomodulatory role for Sap2p on C. albicans was also observed, it appeared unlikely that $\mathrm{p} 43$ activities previously described were caused by Sap2p alone [105]. Nevertheless, this study assessed the potential of an intradermal (i.d.) vaccination with $10 \mu \mathrm{g}$ Sap2p inoculum incorporated in alum adjuvant in preventing systemic candidiasis in BALB/c mice. After a lethal inoculum of $C$. albicans an effective protection was obtained in Sap2p immunized mice with a survival rate of around $75 \%$. The immune protection against systemic candidiasis in mice immunized with Sap $2 p$ is antibody-mediated as indicated by the correlation of survival with an increase in serum antibodies to Sap2p, and by the passive transfer of anti-Sap2 IgG that significantly decreased yeast burden in kidneys of $C$. albicans-infected mice.

Meanwhile, intravaginal (i.v.g) and intranasal (i.n.) immunizations with a Sap preparation, mainly consisting of Sap2p, showed that neutralization of these secreted proteins could also be effective in conferring protection against vaginal candidiasis in the rat model. An inoculum of $100 \mu \mathrm{g}$ of Sap with cholera toxin as adjuvant induced a high degree of protection against vaginal challenge with $C$. albicans. This study showed that the i.n. route is as efficient as the i.v.g. in inducing protection against mucosal candidiasis and it is recognized that i.n. route is much more easy to subject compliance to self administration [106]. In order to specify the antigen to propose a candidate vaccine against mucosal candidiasis, a recombinant and truncated Sap2 protein $(\mathrm{rSap} 2 \mathrm{t})$ was produced and the immunization with this recombinant protein induced antibody-mediated protection against $\mathrm{Can}$ - 
dida infection in an experimental model of rat vaginitis, confirming the previous results [107].

Hyrlp is an important virulence factor for C. albicans, a mannoprotein on the cell wall implicated in the resistance to phagocyte killing and another approach to induce protection used this protein [108]. Mice were immunized with a recombinant fragment (rHyr1p-N) in complete Freund's adjuvant or in Alhydrogel and then infected with $C$. albicans. The results showed that vaccination with $\mathrm{rHyr} 1 \mathrm{p}-\mathrm{N}$ mixed with both adjuvants markedly improved survival in a range of $60 \%$ or $70 \%$. The mechanism of resistance included the action of polyclonal antibodies specific to anti-rHyrlp that enhanced mouse neutrophil killing activity evidenced by the lower organ fungal burden [108]. In order to expand these findings to both immunocompetent and neutropenic mice, and to evaluate if this vaccination strategy also protects against non-albicans Candida spp, immunizations with $\mathrm{rHyr} 1 \mathrm{p}-\mathrm{N}$ in alum were tested. Results showed a dose dependent improved survival rates in immunocompetent mice compared to mice receiving adjuvant alone, with the best results observed with $33 \mu \mathrm{g}$ of rHyr $1 \mathrm{p}-\mathrm{N}$ in alum (survival rate of around $40 \%$ ). Vaccination with the highest dose also reduced the tissue fungal burden by approximately 16 -fold when compared to control mice. This vaccine effectively protected neutropenic mice against candidiasis (survival rate of around 20\%) and significantly increased the mouse immune response as determined by detection of increased anti-rHyrlp-N antibody titers and reduced CFUs in kidneys. The rHyrlp-N vaccine also induced a significant reduction of tissue fungal burden of vaccinated mice and then infected with $C$. glabrata, $C$. parapsilosis and $C$. tropicalis. The mechanism of protection rendered by $\mathrm{rHyr} 1 \mathrm{p}-\mathrm{N}$ appears to be attributed, at least in part, to protective antibody response as evidenced by the results obtained with passive immunization with purified rabbit anti-Hyr $1 \mathrm{p}$ IgG prolonged survival of mice infected with C. albicans.

Evidence has accumulated that antibodies specific for certain yeast cell surface epitopes may be protective and thus, screens for antibodies against those epitopes expressed during pathogenesis in human disseminated candidiasis have been performed [59]. From these studies, some of the antigens responsible for the induction of antibodies identified during the convalescent-phase or that could be correlated with higher probability of recovery were tested. Anti-wall enolase antibodies stand out as protective antibody and thus enolase was tested to evaluate if it would induce protection against a systemic infection in the mice model. $100 \mu \mathrm{g}$ of purified recombinant enolase (rEno1p) in complete Freund's adjuvant were evaluated in an immunization protocol and results indicated to be effective in reducing the CFUs of organs of immunized mice [109]. The immune protection appeared to be mainly antibody-mediated as indicated by the increased titres of enolase-specific IgG1 and IgG2a in serum of immunized mice that enhanced opsonization mediated yeast killing by neutrophils. Passive transfer of anti-rEno1p also reduced fungal burden of non-immunized infected mice.

Antibodies against heat-shock proteins, particularly anti-Hsp90p, have also been detected in sera from patients that recovered from invasive candidiasis in 
contrast to those that did not survived and consequently Hsp90p, a cell wall protein, has been suggested as an important target for protective studies. In addition, Mycograb (Neu Tec Pharma) a monoclonal antibody binds to the immunodominant epitope of $C$. albicans Hsp90p has been applied in combined therapies with positive results [63]. Two strategies have been exploited, an Hsp90-expressing DNA vaccine and a recombinant Hsp90 protein vaccine [110, 111]. However, and although particularly the recombinant protein induced significant increases in both serum and vaginal hsp90-CA-specific IgG and IgA antibodies when compared to the control group these vaccination approaches did not elicit sufficient protection.

In sum, the best protective effects observed to date have been stimulated by immunization with viable cells from virulent or avirulent $C$. albicans strains. Killed cells or subcellular components have been moderately successful and even less efficient in subunit vaccines. However, in the last years the focus on the development of fungal vaccines has been placed on subunit vaccines that are easily controlled in terms of preparation and composition, enabling a more precise evaluation of their mechanism of action and good manufacturing practices. Although expectations for these vaccines are high, some authors think that for invasive candidiasis univalent vaccines are unlikely to be successful [64]. The reasons for these disbelieve are mainly due to the fact that the most effective vaccines developed are against known virulence factors with inducing of protective antibodies. One of the main characteristic features of $C$. albicans is its extraordinary range of virulence factors that are expressed by families of genes. Most of these gene families are composed of at least ten different members that in their action facilitate tissue invasion by enabling the fungus to escape from, modulate or even exacerbate host immunity, and in most situations play redundant roles. Thus, the univalent vaccines confer a considerable probability that the fungus can escape from immune responses induced by the vaccination strategy by compensatory expression of other virulence traits. Taking into account these observations, the use of at least two unrelated virulence associated fungal immunogens was recommended to be present in the vaccine formulation, such as the Als $3 p$ and the Sap $2 p$.

Other approach to reduce the chances of immune evasion is to go back to the use of a more complex immunization antigen. A recent study used dithiothreitol (DTT) extracted $C$. albicans cell wall surface proteins (CWSP) as antigens incorporated into cationic liposomes as adjuvant/ delivery system. The cationic surfactant dioctadecyldimethylammonium bromide (DODAB) and monoolein, 1monooleoyl-rac-glycerol (MO) were used to develop a DODAB:MO lipid based delivery system loaded with CWSP and determined its immunogenicity for the development of a vaccine against systemic Candida infection [112]. Results showed that immunized mice displayed strong humoral response, with high levels of IgGs against specific cell wall proteins, and re-stimulated splenocytes derived from mice immunized secreted high levels of IFN- $\gamma$ and IL-17. This vaccine strategy was able to protect around $70 \%$ of mice [113]. The main advantage of this system is that liposomes, as particulate carriers, entrap and protect the specific 
protein(s) against degradation, enhancing APC uptake of the liposomes and activation.

Many positive results have been achieved in the last years in the development of fungal vaccines. Technological developments have allowed for a rapid expansion of the number of general strategies for making new vaccines and studies will continue, based on more comprehensive understanding of the immunobiology of pathogen, the type and specificity of immune response required for persistent protection against disease, the attainment of mucosal immunity and the optimal vaccination strategy to achieve protection. From all these studies, several candidate vaccines have been proposed and an important outcome is that the selection of antigens and /or the complexation method of conjugation are critical since it may result in the development of potentially useful protective vaccine candidates or it may enhance disease susceptibility. Problems associated with conjugated vaccines such as, variation in antigen loading, immunogenic linkers, and antigenic carrier proteins must be considered. The important point, from the standpoint of antibody-mediated protection, is that the enormous antigenic complexity of the Candida cell should not be expected to reliably induce production of protective antibodies, as defined by specificity, titer, isotype and effector function. Candida albicans antigens that appeared to have potential immunoprotective responses seem to be proteins or glycoproteins components that play their structural/physiological role outside the plasma membrane, belonging to the cell wall, or that are secreted to the exocellular environment.

In recent years, advancement of vaccines has focused on improving immuneadjuvant and the use of novel vaccine carriers. Some of these particulate carriers also show adjuvant properties. Herein, we will present the adjuvant carriers that are used in commercial vaccines, and the carriers that are being used in the development of fungal vaccines.

\subsection{Licensed adjuvants carriers}

The main goal of vaccination is the induction of a protective immunity against a particular disease, and in some subunit vaccines this can only be achieved by addition of an adjuvant or adjuvant combinations. Sub-unit, highly purified antigens may lack PAMPs needed to activate the innate immune system and downstream adaptive responses. It is thought that the use of adjuvants (Latin word adjuvare, meaning "to help or aid") may help to overcome this first step on the innate immune response [114]. Thus, the choice of adjuvant/s is important since it can direct the type of adaptive immune response to the administered antigen, preferentially activating specific T-cell responses. Adjuvants may also improve immune responses in populations where responses to vaccines are typically reduced, such as infants, the elderly and the immunocompromised, which is the more vulnerable population for acquiring fungal infections. 
Although adjuvants are important components of the vaccine strategy, their mechanisms of action only recently are being revealed [115]. Latest evidence suggest that adjuvants may have one or more of the following mechanisms of action: (1) depot effect (sustained release of antigen at the site of injection), (2) cellular recruitment at the site of injection, (3) increase antigen uptake and presentation to APCs, (4) up-regulation of MHC II and co-stimulatory molecules and migration to the draining lymph nodes, (5) up-regulation of cytokines and chemokines, and (6) activation of inflammasomes [115]. The most widely recognized vaccine adjuvants are aluminium based mineral salts, emulsions, virosomes, and liposomes, which are also considered delivery systems.

The most well known adjuvants in vaccination strategies are aluminium based mineral salts, also known as alum. Aluminium salts consist of crystalline nanoparticles that aggregate to form a heterogeneous dispersion of particles of several microns. They are highly charged and conducive, which allows the binding between the antigens or immunomodulatory molecules to alum due to strong electrostatic interactions $[116,117]$. Regardless the progress in understanding aluminium salts its mode of action is still poorly understood. It is described that in the peritoneal cavity of mice alum induces recruitment of immune cells through induction of chemoattractants like CCL2 the neutrophil chemotaxin KC (CXCL1) and eosinophil chemotaxin eotaxin (CCL11) [118]. Eosinophils appear to have a crucial role in priming and differentiation of B cells, resulting in robust antibody production and Th2-type immune responses [119]. However, there is evidence suggesting that injection of alum leads to tissue damage and cell death with release of endogenous danger signals and inflamassome activation, which leads to the release of IL-1 $\beta$, IL-18, in a caspase- 1 dependent manner [120]. There are some conflicting results with regard to the role of inflammasomes in adjuvant activity of alum but the most recent idea is that inflammasomes activation by alum might play an important role in activating innate immunity, but the contribution of inflammasomes in activation of adaptive immunity remains unclear [119]. Aluminum adjuvants act primarily to increase antibody production and are therefore suitable for vaccines targeting pathogens in which the antibodies mediated killing is important, therefore may not be suitable for vaccine strategies where a strong cellular immunity is essential.

An emulsion is a mixture of two non-miscible liquids and an emulsifier that is required to stabilize the emulsion by increasing its kinetic stability. Depending on the water-to-oil ratio, the type of emulsifier, and the mixing procedure, a water-inoil (W/O) or an oil-in-water (O/W) emulsion may be produced [121]. The MF59 was the first oil-in-water adjuvant to be developed and approved for use in human vaccines and it is the second most frequently used in licensed vaccines after alum. It is composed by squalene, polysorbate 80 and sorbitan trioleate. Similar to alum, MF59 also induces the release of the chemokines such as CCL2, CCL3, CCL4 and CXCL8, which are involved in cell recruitment from blood into peripheral tissue. MF59 also accelerates and enhances monocyte differentiation into DCs, augments 
Ag uptake, and facilitates migration of DCs into tissue-draining lymph nodes to prime adaptive immune responses [122]. MF59 does not seem to be correlated with enhanced antibodies titers and it is seems to induce balanced Th1/Th2 responses [115].

Freund's adjuvants were the earlier used emulsion adjuvants. Freund's complete adjuvant (FCA) is a mixture of $85 \%$ mineral oil (Marco 52) and $15 \%$ emulsifier (Arlacel A, mannide monooleate) prepared with $500 \mu \mathrm{g} / \mathrm{ml}$ of heat-killed and dried Mycobacterium tuberculosis. Incomplete Freund's adjuvant (IFA) lacks the mycobacterial component, making it safer but less potent. However, Freund's adjuvants were considered too reactogenic for continued use in humans [123].

More recently two new emulsions, Adjuvants Systems 03 (AS03) and 04 (AS04), were developed. AS03 is composed of squalene, $\alpha$-tocopherol and polysorbate 80, while adjuvant AS04 is composed of monophosphoryl lipid A preparation (MPL) and aluminum salt. AS04 was shown to induce optimal immune responses only when co-localized with antigen, providing a depot effect [124]. The depot effect at the injection site is the most widely recognized mechanism of action of adjuvants. This slow release of antigens ensures constant stimulation of the immune system and has been correlated with production of high antibody titers. Recent evidence indicates that this is not the main mechanism of action of alum or MF59. AS04 was also found to induce maturation of DCs (via TLR4), which then trafficks to the draining lymph nodes to activate antigen-specific T cells [124]. AS03 was found to up-regulate cytokines, such as colony-stimulating factor 3 (CSF3) and IL-6 and chemokines as CCL2, CCL3, and CCL5, which then leads to cellular recruitment at the injection. The mononuclear cells after taking up antigen traffick to draining lymph nodes [125]. These data suggest that the direct effects of AS04 and AS03 adjuvants are on innate immune cells and effectors, activating of APCs and trafficking to draining lymph node, and also inducing secretion of cytokines and chemokines. It is described that these effects will enhance antibody responses and Th1 responses $[115,124,125]$.

Virosomes are spherical unilamellar vesicles of phospholipid carrying envelope virus proteins on their surface or encapsulated within the lumen [126]. Influenza virosomes have been used in the first vaccine to use an adjuvant other than aluminum, a vaccine against hepatitis A licensed in 1994 [126]. Influenza virosomes ensures robust and long-lasting immune responses against subunit antigens with an excellent safety profile. The adjuvant effect of virosomes is once more related to their ability to enhance antigen processing and presentation by APCs. Through fusion of the virosome membrane with the membrane of endosomes or phagosomes the encapsulated protein antigens gain direct access to the MHC class I presentation pathway [127]. In addition, virossomes can also deliver their contents to DCs, MHC class I and class II molecules and induce maturation of DCs, being able to activate $\mathrm{T}$ helper cells [128]. This phenomenon of presentation of antigen located in the MHC-class II pathway to MHC-class I pathway is called "cross presentation" and helps the development of both $\mathrm{T}$ helper and $\mathrm{T}$ cytotoxic responses, 
against the antigen [129]. This cross-presentation has been considered a major focus to the success of producing an effective immune response to a vaccine, particularly against intracellular parasites. The cytokine profile induced by virosomes, including TNF- $\alpha$, GM-CSF, IFN- $\gamma$ and IL-2, is consistent with a Th1 response.

Liposomes are double or multilayered, biodegradable carrier vesicles made up of phospholipids and/or synthetic lipids that consist of a lipid bilayer shell with an aqueous core. Liposomal formulations can be tailored in terms of the lipid composition, the liposomal charge, the size/lamellarity and/or the membrane fluidity in order to obtain optimal retention of different types of antigens, including adjuvants, and enhancement of the desired vaccine-specific immune responses [130]. The conjugation of hydrophilic molecules in the surface of liposomes is also possible and further facilitates the uptake by phagocytes [131]. In addition, liposomes are highly acceptable by the human body and present low toxicity. From these characteristics liposomes are, without any doubt, the most studied carrier system and the interest for liposome-based vaccines has markedly increased over the last decades.

The lipids selected for liposomal system will ultimately determine their properties and ability of inducing maturation of DCs, which is important to achieve an optimized vaccine carrier. The particle size governs cellular trafficking to secondary lymph nodes, antigen uptake, and cellular responses and it has been described that small particles $(20-200 \mathrm{~nm})$ drain freely towards lymph nodes, while large particles $(500,1000 \mathrm{~nm})$ are dependent on DCs for transport to the lymph nodes [132]. The selection of the type of lipids also determines liposomal membrane fluidity, which is linked to cellular trafficking and antigen presentation to APCs. It has been determined that rigid, saturated dimethyl dioctadecyl ammonium (DDA) lipid greatly enhances priming ability of Th1-directed immune response, compared with a fluid unsaturated lipid [133]. The surface charge of the liposome, the zeta potential value, is also determined by their lipid composition and dictates the colloidal stability of the formulation. The surface charge of liposomes has a major importance in the immune response, with cationic liposomes having an advantage over their neutral and anionic counterparts [131]. Indeed, the high surface density of positive charges offers an important platform for antigen adsorption due to electrostatic interactions. Moreover, the interaction between the positively charged liposomes and the negatively charged membranes of APCs facilitates liposome uptake, increasing the vaccine potency without the need to increase the concentration of antigen [134]. In addition, the depot effect at the site of injection has also been described for the cationic adjuvant formulation (CAF) 01, a combination of DDA / TDB (trehalose-6,6-dibehenate) which is currently in phase I clinical trial $[115,135]$.

In sum, an essential point in the investigation of modern adjuvants is not only the initiation of a potent adequate immune response but mainly targeting APCs receptors to achieve such response avoiding as much as possible the toxicity associ- 
ated. Moreover, the ideal adjuvant should be able to promote an antigen-specific immune response, and should be nontoxic, biocompatible, readily biodegraded and eliminated, inexpensive to produce, stable before administration, and physicochemically well defined to facilitate quality control important to ensure reproducible manufacturing and activity.

\subsection{Vaccine strategies against Candida infections that advanced to clinical} trails

Many positive results have been achieved in the development of fungal vaccines in the last years. However, few vaccine formulations directed against $C$. albicans have successfully completed Phase I clinical trials, and although the majority of the studies are concerned with the development of vaccines against systemic candidiasis the only approaches that enter clinical trails were against recurrent vulvovaginal candidiasis (VVC). The first study described in the literature is the Phase II study of D.651, an oral vaccine designed to prevent VVC prepared with C. albicans ribosomes and membrane proteoglycan from a nonencapsulated Klebsiella pneumoniae as adjuvant [136]. Latter, the PEV-7 from Pevion Biotech (Ittigen, Switzerland) composed of an antigen fragment of Sap $2 \mathrm{p}$ embedded in a virosomal formulation with intrinsic adjuvanticity was proposed against VVC infection. Finally, the NDV-3 from NovaDigm Therapeutics (USA) based on a recombinant protein fragment from Als3 with alum as the adjuvant is also in Phase $1 \mathrm{~b} / 2$ a clinical trial to evaluate NDV-3 again in patients diagnosed with VVC.

The preliminary phase II study of D.651 vaccine was conducted in 22 women with a history of frequent recurrences of vulvovaginal candidiasis. Vaccine was taken orally and administered intermittently over six months in capsules. The vaccine was well tolerated and reduced an average of 3.59 attacks of VVC per 6 months to and average of 0.55 attacks per 6 months. However, this trial was conducted without placebo and no further studies on this vaccine have been documented.

The PEV7 vaccine is based on a truncated and enzymatically inactive recombinant aspartyl proteinase-2 (rSap2) of $C$. albicans presented on the surface of influenza virosomes [106, 107, 137]. PEV7 was considered to be safe in a repeateddose toxicological study in rats and generated a robust serum IgG and IgA antiSap2 antibody response in mouse and rat models after intramuscular and intravaginal immunizations. Phase I clinical trial used lyophilized virosome formulations for intramuscular and intravaginal application with the intention to assess the safety and immunogenicity of PEV7 via a systemic prime/mucosal boost regime in 48 healthy volunteers. Half of the women received intramuscular injections, while the other half received intravaginal capsules. This capsular formulation for intravaginal application represents an innovation with the advantage of an easier administration, favoring patient compliance. Results showed that $100 \%$ of the vaccinated women developed specific and functional B cell memory. Both routes of vaccination induced a rapid and specific production of antibodies either in serum and/or 
cervicovaginal secretion, which was very encouraging with regards to the therapeutic potential of the vaccine [138]. A summary of the study can be found at clinicaltrials.gov website, identifier number NCT01067131.

The NDV-3 trial is a multi-center, double-blind, randomized, placebocontrolled study to evaluate the safety, tolerability, immunogenicity and efficacy of this vaccine. Due to previous results, this vaccine was developed to treat and prevent infections caused by Candida but also to Staphylococcus aureus, being the first vaccine to demonstrate preclinical cross-kingdom potential [103]. This Phase $1 \mathrm{~b} / 2 \mathrm{a}$ trial involved 188 patients from multiple centers in the United States with the aim to study and estimate the effect of a single, intramuscularly-administered dose of NDV-3, as compared to placebo, by evaluating safety and tolerability, as well as humoral and cellular immune responses. The study will also summarize recurrence of VVC over six- and 12-month period, time-to-onset of first VVC episode and severity of subsequent VVC episodes. A summary of the study can be found at clinicaltrials.gov website, identifier number NCT01926028.

\subsection{Concerns to overcome to further advance in fungal vaccines}

The development of innovative vaccines to protect patients from fungal and bacterial infections that can be recurrent, drug-resistant, and in some cases lifethreatening are still necessary. Other vaccines are ready to enter clinical trials if industrial interest and sufficient monetary support can be raised. Nevertheless, there are still some crucial points to solve such as: (1) the majority of the studies were performed in animal models that have limitations, (2) further understanding of the mechanisms of the $C$. albicans-host interaction in mucosal and systemic infections, (3) the development of autoimmunity.

The majority of the studies have been developed in animal models, that although very useful do not fully recapitulate infection by the opportunistic commensal $C$. albicans. Mice and humans are undoubtedly substantially different regarding their innate immune response to Candida. One of the main limitations is that, unlike humans, mice do not have a C. albicans GI flora and they lack Candida serum antibodies. Several attempts to develop a more real model have been made, and there has been indications that Candida-colonized mice were able to developed fungal-specific antibodies in the serum due to the colonization and that, these animals if immunized with a specific protein, were able to develop specific antibodies to that immunogen [139]. However, the information that can be gained from using such mice is still limited. The consequences may be that vaccines that are very efficient in the animal model could be less effective in human, and the protection of the vaccine is not persistent or high-grade or cause unacceptable toxicity. Thus, in preclinical trials the effectiveness of vaccines should be tested in different species of animals, including mice, rabbits and monkeys. The infection models could include immune-normal animals, immune-deficient animals, and could involve variations in the symbiotic bacteria.

To design any vaccine the mechanisms that confer protective immunity in the host against fungi must be fully understood. This knowledge is important in the 
selection for constituents from the fungus that elicit the particular type of immune response. In addition, there is little control of the $C$. albicans strain used in the studies, the dose of the inocula to produce the intended outcome of death or tissue infection, the dosage and delivery time of the vaccines to produce the greatest protection effect, information regarding the different outcomes form various routes of Candida infection, or the multiple parameters tested, such as tissue CFU and survival rates. This is further complicated in the development of a multivalent vaccine. Although substantial advances have been made in the understanding of hostCandida interactions, the current paradigm of $C$. albicans-specific immunity suggests that pathogenic growth of the fungus is prevented by a combination of innate and adaptive Th1/Th17 responses, which activates phagocytes as the major candidacidal effectors $[33,58]$. However, despite the idea that Th17 cells are protective against fungal infections, recent studies suggest that inflammatory Th17 and Treg responses are protective at mucosal surfaces but in systemic candidiasis a time dependent and coordinated Th17/Treg response is fundamental for a positive outcome [63]. The balance between protective versus pathogenic immunity is crucial in determining the disease outcome. This is one of the reasons that justify why vaccines against mucosal candidiasis have advanced to clinical trails. Another concern in the development of subunit vaccines is the adjuvant used. Adjuvants have been used for long time but their mechanisms of action are not fully understood. They may act by a combination of various mechanisms to create a local immuno-competent environment at the injection site. Many of the preclinical studies were conducted using adjuvants that are not recommended for human use such as the Freund's adjuvant, and when switched to an adjuvant recommended the results were not the same, and in many cases were lower than expected. In addition, and probably due to the lack of knowledge about their mechanism of action, adjuvants are selected just taking into consideration what is in use. Adjuvants should be selected depending on the type of innate responses that are needed to be activated, because they may alter the quality and quantity of adaptive immune responses. That is why just changing the adjuvant without considering its mechanism of action may not produce the expected results. Thus, more studies should be developed in order to better understand the mechanisms of action of adjuvants, providing critical information that will help in a rational design of vaccines and inform on adjuvant safety. These informations will lead to the recommendation of new adjuvants for human use.

The development of autoimunity was always an important concern, particularly in the case of commensal species as $C$. albicans. Keeping the balance of the immune system in eliminating invading pathogens, while still maintaining selftolerance to avoid autoimmunity, is critical for the body's health. In this balance, the mucosa microbiota provides benefits to the host by actively participating in the regulation of immune homeostasis. These evidences are confirmed by the consequences of altering the gut microbial communities, termed dysbiosis, such as the autoimmune disorders. The mucosa microbiota has a profound effect on both the 
innate and adaptive immune system so it is not surprising that some members of the microbiota have been linked to autoimmune diseases. It has been determined that the microflora of mice with colitis fails to metabolize tryptophan into metabolites that act as aryl hydrocarbon receptor (AHR) ligands, modulating production of IL-22, an important cytokine for intestinal homeostasis [140]. Intestinal inflammation is attenuated after inoculation of mice with three Lactobacillus strains capable of metabolizing tryptophan or by treatment with an AHR agonist. Despite difficulties in fungal DNA extraction methods and fungal identification methods, the gut, oral, lung and skin niches presented four genera in common: Aspergillus, Candida, Cryptococcus, and Penicillium [141]. Fungi have long been suspected to be involved in inflammatory bowel disease (IBD) pathogenesis since many genes involved in the immune response against fungi have associated single nucleotide polymorphisms (SNPs) in IBD patients, such as in DECTIN-1, CARD9 or NFkBp105 genes in the signaling pathways of antigen-presenting cells, and IL12p40, IL23R or IL-6ST, cytokines and cytokine receptors involved in Th17 and Th1 polarization, among others [142]. These findings suggest a role of fungi in inflammatory pathogenesis. Thus, it is not surprising to find that fungi, and particularly C. albicans, is more abundant in the gut microbiota of these patients. But this enhancement in the fungal population is particularly related to a genetic defect in microbial sensing in the susceptible human population. Using animal models, gut inflammation induced by dextran sodium sulfate (DSS) promoted C. albicans growth and this growth enhances inflammation. Thus, it seems that there is a vicious circle in which intestinal inflammation induces $C$. albicans proliferation, which itself enhances the inflammatory process. That is why it is described that $C$. albicans has a negative impact on inflammation. Thus, any factor that significantly alters the local microbiota such as prolonged use of broad-spectrum antibiotics, enhances the fungal population in the microbiota and augments intestinal inflammation, contributing to the initiation of the vicious circle and enhancing the changes of fungi translocation and systemic infection. But this predisposition to increased inflammation may not be a direct action of Candida in inducing the autoimmune dysfunction, unless patients present genetic defect in fungi sensing. Indeed, host genes affect the composition and function of the gut microbiota.

In order to induce an autoimmune dysfunction, vaccination against $C$. albicans either enhances chronic inflammation, particularly at the mucosa, or develops an adaptive response against an epitope similar to a human molecule. Antibodies against Saccharomyces cerevisiae mannan as well as against other fungal cell wall components, such as anti-laminarin that identifies $\beta$-1,3-glucans and against $\beta$ 1,4-N-acetylglucosamine, present in the chitin layers, have been shown to be associated with Crohn's disease [141, 143]. But once more, the existence of these antibodies may be due to the fact that in susceptible individuals intestinal inflammation induces $C$. albicans proliferation, enhancing the changes of fungi translocation and being seen by the immune system. There is no evidence that in normal individuals the presence of these antibodies enhance the probability of mucosa inflammation. On the contrary, from the vaccination studies against Can- 
dida vaginal infections, a reduction in the inflammation sign as well as a local reduced fungal burden is documented [144]. However, regarding vaccine development studies against systemic candidiasis mucosal inflammation is not a parameter that is frequently included in these studies. Thus, the study of the induction of inflammatory at the mucosa should also be included in the studies addressing systemic candidiasis.

In terms of cross-reactions responses against molecules/epitopes with homology to human molecules, epitope mapping analysis has showed that some heat shock proteins epitopes from $C$. albicans may have as much as $100 \%$ direct homology with human heat shock proteins [76]. Thus, antibodies developed against the heat shock proteins, particularly Hsp90p raise the concern of development of autoimmune reactions. In children idiopathic short stature with $C$. albicans and/or $H$. pylori gut colonization it was reported that the incidence of autoantibodies against selected neuropeptides is high due to molecular mimicry between antigens of these microbiota and the selected neuropeptides [145]. However, further studies are necessary to elucidate this issue. To avoid induction of these autoantibodies by vaccination the selection of target molecules is essential, molecules that are unique to the fungi should be addressed and an epitope mapping analysis should always be performed.

In summary, despite the advances in our knowledge and understanding in pathogenesis and immune responses, invasive fungal infections continue to result in significant morbidity and mortality in critically ill patients. The traditional antifungal chemotherapy has met some limitations, such as the toxicity and emergence of resistance, limiting their effectiveness. Therefore, there is an urgent need to improve treatment options for these patients and vaccines can represent novel approaches against fungal infections. Although the currently available vaccines have demonstrated good protection, there still a long way to go to before a vaccine to be applied in patients with predisposing factors to systemic fungal infections will be achieved.

\section{Bibliografy}

1. Gow, N.A., et al., Candida albicans morphogenesis and host defence: discriminating invasion from colonization. Nat Rev Microbiol, 2012. 10(2): p. 112-22.

2. Mayer, F.L., D. Wilson, and B. Hube, Candida albicans pathogenicity mechanisms. Virulence, 2013.4(2): p. 119-28.

3. Eberl, G., A new vision of immunity: homeostasis of the superorganism. Mucosal Immunol, 2010. 3(5): p. 450-60. 
4. Paramythiotou, E., et al., Invasive fungal infections in the ICU: how to approach, how to treat. Molecules, 2014. 19(1): p. 1085-119.

5. Kullberg, B.J. and M.C. Arendrup, Invasive Candidiasis. N Engl J Med, 2015. 373(15): p. 1445-56.

6. Eggimann, P. and D. Pittet, Candida colonization index and subsequent infection in critically ill surgical patients: 20 years later. Intensive Care Med, 2014. 40(10): p. 1429-48.

7. Kett, D.H., et al., Candida bloodstream infections in intensive care units: analysis of the extended prevalence of infection in intensive care unit study. Crit Care Med, 2011.39(4): p. 665-70.

8. Sampaio, P., et al., Virulence attenuation of Candida albicans genetic variants isolated from a patient with a recurrent bloodstream infection. PLoS One, 2010.5(4): p. e10155.

9. Hirakawa, M.P., et al., Genetic and phenotypic intra-species variation in Candida albicans. Genome Res, 2015. 25(3): p. 413-25.

10. Becker, K.L., et al., Antifungal innate immunity: recognition and inflammatory networks. Semin Immunopathol, 2015. 37(2): p. 107-16.

11. Cheng, S.C., et al., Interplay between Candida albicans and the mammalian innate host defense. Infect Immun, 2012. 80(4): p. 1304-13.

12. De Luca, A., et al., IL-22 defines a novel immune pathway of antifungal resistance. Mucosal Immunol, 2010. 3(4): p. 361-73.

13. Duhring, S., et al., Host-pathogen interactions between the human innate immune system and Candida albicans-understanding and modeling defense and evasion strategies. Front Microbiol, 2015. 6: p. 625.

14. Netea, M.G., et al., An integrated model of the recognition of Candida albicans by the innate immune system. Nat Rev Microbiol, 2008. 6(1): p. 67-78.

15. Netea, M.G., et al., Immune defence against Candida fungal infections. Nat Rev Immunol, 2015. 15(10): p. 630-42.

16. Romani, L., Immunity to fungal infections. Nat Rev Immunol, 2011. 11(4): p. 275-88.

17. Naglik, J.R. and D. Moyes, Epithelial cell innate response to Candida albicans. Adv Dent Res, 2011. 23(1): p. 50-5.

18. Luo, S., et al., Complement and innate immune evasion strategies of the human pathogenic fungus Candida albicans. Mol Immunol, 2013. 56(3): p. 161-9.

19. Lievin-Le Moal, V. and A.L. Servin, The front line of enteric host defense against unwelcome intrusion of harmful microorganisms: mucins, antimicrobial peptides, and microbiota. Clin Microbiol Rev, 2006. 19(2): p. 315-37.

20. Johansson, M.E., et al., The inner of the two Muc2 mucin-dependent mucus layers in colon is devoid of bacteria. Proc Natl Acad Sci U S A, 2008. 105(39): p. 15064-9. 
21. Kavanaugh, N.L., et al., Mucins suppress virulence traits of Candida albicans. MBio, 2014. 5(6): p. e01911.

22. Bobek, L.A. and H. Situ, MUC7 20-Mer: investigation of antimicrobial activity, secondary structure, and possible mechanism of antifungal action. Antimicrob Agents Chemother, 2003. 47(2): p. 643-52.

23. Fukata, M. and M. Arditi, The role of pattern recognition receptors in intestinal inflammation. Mucosal Immunol, 2013. 6(3): p. 451-63.

24. Netea, M.G., et al., Immune sensing of Candida albicans requires cooperative recognition of mannans and glucans by lectin and Toll-like receptors. J Clin Invest, 2006. 116(6): p. 1642-50.

25. Weindl, G., et al., Human epithelial cells establish direct antifungal defense through TLR4-mediated signaling. J Clin Invest, 2007. 117(12): p. 3664-72.

26. Cohen-Kedar, S., et al., Human intestinal epithelial cells respond to betaglucans via Dectin-1 and Syk. Eur J Immunol, 2014. 44(12): p. 3729-40.

27. Sun, W.K., et al., Dectin-1 is inducible and plays a crucial role in Aspergillus-induced innate immune responses in human bronchial epithelial cells. Eur J Clin Microbiol Infect Dis, 2012. 31(10): p. 275564.

28. McKenzie, C.G., et al., Contribution of Candida albicans cell wall components to recognition by and escape from murine macrophages. Infect Immun, 2010. 78(4): p. 1650-8.

29. Vance, R.E., R.R. Isberg, and D.A. Portnoy, Patterns of pathogenesis: discrimination of pathogenic and nonpathogenic microbes by the innate immune system. Cell Host Microbe, 2009. 6(1): p. 10-21.

30. Li, M., et al., The expression of beta-defensin-2, 3 and LL-37 induced by Candida albicans phospholipomannan in human keratinocytes. J Dermatol Sci, 2011. 61(1): p. 72-5.

31. Moyes, D.L., et al., A biphasic innate immune MAPK response discriminates between the yeast and hyphal forms of Candida albicans in epithelial cells. Cell Host Microbe, 2010. 8(3): p. 225-35.

32. Cheng, S.C., et al., The dectin-1/inflammasome pathway is responsible for the induction of protective T-helper 17 responses that discriminate between yeasts and hyphae of Candida albicans. J Leukoc Biol, 2011. 90(2): p. 357-66.

33. Hornby, J.M., et al., Quorum sensing in the dimorphic fungus Candida albicans is mediated by farnesol. Appl Environ Microbiol, 2001. 67(7): p. 2982-92.

34. Lowman, D.W., et al., Novel structural features in Candida albicans hyphal glucan provide a basis for differential innate immune recognition of hyphae versus yeast. J Biol Chem, 2014. 289(6): p. 3432-43.

35. Bain, J.M., et al., Non-lytic expulsion/exocytosis of Candida albicans from macrophages. Fungal Genet Biol, 2012.49(9): p. 677-8. 
36. Filler, S.G. and D.C. Sheppard, Fungal invasion of normally nonphagocytic host cells. PLoS Pathog, 2006. 2(12): p. e129.

37. Mech, F., et al., Epithelial invasion outcompetes hypha development during Candida albicans infection as revealed by an image-based systems biology approach. Cytometry A, 2014. 85(2): p. 126-39.

38. Wilson, D., et al., Identifying infection-associated genes of Candida albicans in the postgenomic era. FEMS Yeast Res, 2009. 9(5): p. 688700.

39. Nahum, A., et al., The biological significance of TLR3 variant, $L 412 F$, in conferring susceptibility to cutaneous candidiasis, CMV and autoimmunity. Autoimmun Rev, 2012. 11(5): p. 341-7.

40. Kasperkovitz, P.V., et al., Toll-like receptor 9 modulates macrophage antifungal effector function during innate recognition of Candida albicans and Saccharomyces cerevisiae. Infect Immun, 2011. 79(12): p. 4858-67.

41. Wagener, J., et al., Fungal chitin dampens inflammation through IL-10 induction mediated by NOD2 and TLR9 activation. PLoS Pathog, 2014. 10(4): p. e1004050.

42. Brown, G.D., et al., Dectin-1 is a major beta-glucan receptor on macrophages. J Exp Med, 2002. 196(3): p. 407-12.

43. Ferwerda, G., et al., Dectin-1 synergizes with TLR2 and TLR4 for cytokine production in human primary monocytes and macrophages. Cell Microbiol, 2008. 10(10): p. 2058-66.

44. Saijo, S., et al., Dectin-2 recognition of alpha-mannans and induction of Th17 cell differentiation is essential for host defense against Candida albicans. Immunity, 2010. 32(5): p. 681-91.

45. Zhu, L.L., et al., C-type lectin receptors Dectin-3 and Dectin-2 form a heterodimeric pattern-recognition receptor for host defense against fungal infection. Immunity, 2013. 39(2): p. 324-34.

46. Koh, A.Y., et al., Mucosal damage and neutropenia are required for Candida albicans dissemination. PLoS Pathog, 2008. 4(2): p. e35.

47. Qian, Q., et al., Elimination of mouse splenic macrophages correlates with increased susceptibility to experimental disseminated candidiasis. $\mathrm{J}$ Immunol, 1994. 152(10): p. 5000-8.

48. Erwig, L.P. and N.A. Gow, Interactions of fungal pathogens with phagocytes. Nat Rev Microbiol, 2016. 14(3): p. 163-76.

49. Aratani, Y., et al., Critical role of myeloperoxidase and nicotinamide adenine dinucleotide phosphate-oxidase in high-burden systemic infection of mice with Candida albicans. J Infect Dis, 2002. 185(12): p. 1833-7.

50. Bain, J.M., et al., Candida albicans hypha formation and mannan masking of beta-glucan inhibit macrophage phagosome maturation. MBio, 2014.5(6): p. e01874. 
51. Zielinski, C.E., et al., Pathogen-induced human TH17 cells produce IFNgamma or IL-10 and are regulated by IL-1beta. Nature, 2012. 484(7395): p. 514-8.

52. Puel, A., et al., Chronic mucocutaneous candidiasis in humans with inborn errors of interleukin-17 immunity. Science, 2011. 332(6025): p. 65-8.

53. Bonifazi, P., et al., Balancing inflammation and tolerance in vivo through dendritic cells by the commensal Candida albicans. Mucosal Immunol, 2009. 2(4): p. 362-74.

54. Huang, W., et al., Requirement of interleukin-17A for systemic antiCandida albicans host defense in mice. J Infect Dis, 2004. 190(3): p. 62431.

55. Whibley, N. and S.L. Gaffen, Brothers in arms: Th17 and Treg responses in Candida albicans immunity. PLoS Pathog, 2014. 10(12): p. e1004456.

56. Ibrahim, A.S., et al., NDV-3 protects mice from vulvovaginal candidiasis through $T$ - and B-cell immune response. Vaccine, 2013. 31(47): p. 554956.

57. Krause, R., et al., Elevated levels of interleukin 17A and kynurenine in candidemic patients, compared with levels in noncandidemic patients in the intensive care unit and those in healthy controls. J Infect Dis, 2015. 211(3): p. 445-51.

58. Whibley, N., et al., Expansion of Foxp3(+) T-cell populations by Candida albicans enhances both Th17-cell responses and fungal dissemination after intravenous challenge. Eur J Immunol, 2014. 44(4): p. 1069-83.

59. Clancy, C.J., et al., Immunoglobulin $G$ responses to a panel of Candida albicans antigens as accurate and early markers for the presence of systemic candidiasis. J Clin Microbiol, 2008. 46(5): p. 1647-54.

60. Nishiya, C.T., et al., Influence of IgG Subclass on Human Antimannan Antibody-Mediated Resistance to Hematogenously Disseminated Candidiasis in Mice. Infect Immun, 2015. 84(2): p. 386-94.

61. Beenhouwer, D.O., et al., Human immunoglobulin G2 (IgG2) and IgG4, but not IgG1 or IgG3, protect mice against Cryptococcus neoformans infection. Infect Immun, 2007. 75(3): p. 1424-35.

62. Pitarch, A., et al., Proteomics-based identification of novel Candida albicans antigens for diagnosis of systemic candidiasis in patients with underlying hematological malignancies. Proteomics, 2004. 4(10): p. 3084-106.

63. Pachl, J., et al., A randomized, blinded, multicenter trial of lipidassociated amphotericin $B$ alone versus in combination with an antibodybased inhibitor of heat shock protein 90 in patients with invasive candidiasis. Clin Infect Dis, 2006. 42(10): p. 1404-13.

64. Cassone, A., Development of vaccines for Candida albicans: fighting a skilled transformer. Nat Rev Microbiol, 2013. 11(12): p. 884-91. 
65. Spellberg, B., Vaccines for invasive fungal infections. F1000 Med Rep, 2011. 3: p. 13.

66. Kintu, K., et al., Feasibility and safety of ALVAC-HIV vCP1521 vaccine in HIV-exposed infants in Uganda: results from the first HIV vaccine trial in infants in Africa. J Acquir Immune Defic Syndr, 2013. 63(1): p. 1-8.

67. Skorka, K., et al., Indirect induction of regulatory T cells accompanies immune responses during peptide vaccination of chronic lymphocytic leukaemia patients. Br J Haematol, 2015.

68. Berglund, A., et al., The response to vaccination against influenza A(H1N1) 2009, seasonal influenza and Streptococcus pneumoniae in adult outpatients with ongoing treatment for cancer with and without rituximab. Acta Oncol, 2014. 53(9): p. 1212-20.

69. Ariza-Heredia, E.J. and R.F. Chemaly, Practical review of immunizations in adult patients with cancer. Hum Vaccin Immunother, 2015. 11(11): p. 2606-14.

70. Centers for Disease, C. and Prevention, Prevention and control of influenza with vaccines: recommendations of the Advisory Committee on Immunization Practices (ACIP)--United States, 2012-13 influenza season. MMWR Morb Mortal Wkly Rep, 2012. 61(32): p. 613-8.

71. Flowers, C.R., et al., Antimicrobial prophylaxis and outpatient management of fever and neutropenia in adults treated for malignancy: American Society of Clinical Oncology clinical practice guideline. J Clin Oncol, 2013.31(6): p. 794-810.

72. Wisplinghoff, H., et al., Nosocomial bloodstream infections in US hospitals: analysis of 24,179 cases from a prospective nationwide surveillance study. Clin Infect Dis, 2004. 39(3): p. 309-17.

73. Pfaller, M.A. and D.J. Diekema, Epidemiology of invasive candidiasis: a persistent public health problem. Clin Microbiol Rev, 2007. 20(1): p. 133-63.

74. Andes, D.R., et al., Impact of treatment strategy on outcomes in patients with candidemia and other forms of invasive candidiasis: a patient-level quantitative review of randomized trials. Clin Infect Dis, 2012. 54(8): p. 1110-22.

75. Matthews, R.C., J.P. Burnie, and S. Tabaqchali, Immunoblot analysis of the serological response in systemic candidosis. Lancet, 1984. 2(84178418): p. 1415-8.

76. Matthews, R.C., et al., Autoantibody to heat-shock protein 90 can mediate protection against systemic candidosis. Immunology, 1991. 74(1): p. 20-4.

77. Matthews, R., S. Hodgetts, and J. Burnie, Preliminary assessment of a human recombinant antibody fragment to hsp90 in murine invasive candidiasis. J Infect Dis, 1995. 171(6): p. 1668-71. 
78. Hurd, R.C. and C.H. Drake, Candida albicans infections in actively and passively immunized animals. Mycopathol Mycol Appl, 1953. 6(4): p. 290-7.

79. Balogh, E., et al., Experimental investigation of immunreactivity against Candida albicans. 1. Reaction of rabbits immunized with Candida albicans and challenged with living Candida albicans. Mykosen, 1971. 14(8): p. 385-92.

80. Bistoni, F., et al., Evidence for macrophage-mediated protection against lethal Candida albicans infection. Infect Immun, 1986. 51(2): p. 668-74.

81. Saville, S.P., et al., Efficacy of a genetically engineered Candida albicans tet-NRG1 strain as an experimental live attenuated vaccine against hematogenously disseminated candidiasis. Clin Vaccine Immunol, 2009. 16(3): p. 430-2.

82. Fernandez-Arenas, E., et al., Contribution of the antibodies response induced by a low virulent Candida albicans strain in protection against systemic candidiasis. Proteomics, 2004. 4(4): p. 1204-15.

83. Tavares, D., P. Ferreira, and M. Arala-Chaves, Increased resistance in $B A L B / c$ mice to reinfection with Candida albicans is due to immunoneutralization of a virulence-associated immunomodulatory protein. Microbiology, 2003. 149(Pt 2): p. 333-9.

84. Mourad, S. and L. Friedman, Passive immunization of mice against Candida albicans. Sabouraudia, 1968. 6(2): p. 103-5.

85. Segal, E., S. Nussbaum, and L. Barr-Nea, Protection against systemic infections with various Candida species elicited by vaccination with Candida albicans ribosomes. Sabouraudia, 1985. 23(4): p. 275-85.

86. Segal, E. and H. Sandovsky-Losica, Experimental vaccination with Candida albicans ribosomes in cyclophosphamide-treated animals. Sabouraudia, 1981. 19(4): p. 267-73.

87. Segal, E., et al., Induction of protection against candidiasis in tumorbearing mice by vaccination with Candida albicans ribosomes. J Med Vet Mycol, 1987. 25(6): p. 355-63.

88. Torosantucci, A., et al., A novel glyco-conjugate vaccine against fungal pathogens. J Exp Med, 2005. 202(5): p. 597-606.

89. Xin, H., et al., Synthetic glycopeptide vaccines combining beta-mannan and peptide epitopes induce protection against candidiasis. Proc Natl Acad Sci U S A, 2008. 105(36): p. 13526-31.

90. Lopez-Ribot, J.L., et al., Antibody response to Candida albicans cell wall antigens. FEMS Immunol Med Microbiol, 2004. 41(3): p. 187-96.

91. Han, Y. and K.Y. Rhew, Comparison of two Candida mannan vaccines: the role of complement in protection against disseminated candidiasis. Arch Pharm Res, 2012. 35(11): p. 2021-7.

92. Han, Y., M.A. Ulrich, and J.E. Cutler, Candida albicans mannan extractprotein conjugates induce a protective immune response against experimental candidiasis. J Infect Dis, 1999. 179(6): p. 1477-84. 
93. Lipinski, T., et al., A beta-mannan trisaccharide conjugate vaccine aids clearance of Candida albicans in immunocompromised rabbits. Vaccine, 2012. 30(44): p. 6263-9.

94. Xin, H., et al., Self-adjuvanting glycopeptide conjugate vaccine against disseminated candidiasis. PLoS One, 2012. 7(4): p. e35106.

95. Cutler, J.E., Defining criteria for anti-mannan antibodies to protect against candidiasis. Curr Mol Med, 2005. 5(4): p. 383-92.

96. Torosantucci, A., et al., Protection by anti-beta-glucan antibodies is associated with restricted beta-1,3 glucan binding specificity and inhibition of fungal growth and adherence. PLoS One, 2009. 4(4): p. e5392.

97. Li, W.J., et al., Adjuvanticity of a recombinant calreticulin fragment in assisting anti-beta-glucan IgG responses in $T$ cell-deficient mice. Clin Vaccine Immunol, 2013. 20(4): p. 582-9.

98. Ibrahim, A.S., et al., Vaccination with recombinant $N$-terminal domain of Als1p improves survival during murine disseminated candidiasis by enhancing cell-mediated, not humoral, immunity. Infect Immun, 2005. 73(2): p. 999-1005.

99. Spellberg, B.J., et al., The anti-Candida albicans vaccine composed of the recombinant $N$ terminus of Als1p reduces fungal burden and improves survival in both immunocompetent and immunocompromised mice. Infect Immun, 2005. 73(9): p. 6191-3.

100. Spellberg, B.J., et al., Efficacy of the anti-Candida rAls3p-N or rAls1p-N vaccines against disseminated and mucosal candidiasis. J Infect Dis, 2006. 194(2): p. 256-60.

101. Ibrahim, A.S., et al., The anti-Candida vaccine based on the recombinant $N$-terminal domain of Als1p is broadly active against disseminated candidiasis. Infect Immun, 2006. 74(5): p. 3039-41.

102. Lin, L., et al., Considerable differences in vaccine immunogenicities and efficacies related to the diluent used for aluminum hydroxide adjuvant. Clin Vaccine Immunol, 2008. 15(3): p. 582-4.

103. Spellberg, B., et al., The antifungal vaccine derived from the recombinant $N$ terminus of Als3p protects mice against the bacterium Staphylococcus aureus. Infect Immun, 2008. 76(10): p. 4574-80.

104. Tavares, D., et al., Immunoprotection against systemic candidiasis in mice. Int Immunol, 1995. 7(5): p. 785-96.

105. Vilanova, M., et al., Protection against systemic candidiasis in mice immunized with secreted aspartic proteinase 2. Immunology, 2004. 111(3): p. 334-42.

106. De Bernardis, F., et al., Intravaginal and intranasal immunizations are equally effective in inducing vaginal antibodies and conferring protection against vaginal candidiasis. Infect Immun, 2002. 70(5): p. 2725-9.

107. Sandini, S., et al., A highly immunogenic recombinant and truncated protein of the secreted aspartic proteases family (rSap2t) of Candida 
albicans as a mucosal anticandidal vaccine. FEMS Immunol Med Microbiol, 2011.62(2): p. 215-24.

108. Luo, G., et al., Candida albicans Hyrlp confers resistance to neutrophil killing and is a potential vaccine target. J Infect Dis, 2010. 201(11): p. 1718-28.

109. Li, W., et al., Immunisation with the glycolytic enzyme enolase confers effective protection against Candida albicans infection in mice. Vaccine, 2011. 29(33): p. 5526-33.

110. Raska, M., et al., Comparison of protective effect of protein and DNA vaccines hsp90 in murine model of systemic candidiasis. Folia Microbiol (Praha), 2005. 50(1): p. 77-82.

111. Raska, M., et al., Systemic and mucosal immunization with Candida albicans hsp90 elicits hsp90-specific humoral response in vaginal mucosa which is further enhanced during experimental vaginal candidiasis. Med Mycol, 2008. 46(5): p. 411-20.

112. Carneiro, C., et al., DODAB:monoolein liposomes containing Candida albicans cell wall surface proteins: a novel adjuvant and delivery system. Eur J Pharm Biopharm, 2015. 89: p. 190-200.

113. Carneiro, C., et al., Protective effect of antigen delivery using monooleinbased liposomes in experimental hematogenously disseminated candidiasis. Acta Biomater, 2016.

114. Coffman, R.L., A. Sher, and R.A. Seder, Vaccine adjuvants: putting innate immunity to work. Immunity, 2010. 33(4): p. 492-503.

115. Awate, S., L.A. Babiuk, and G. Mutwiri, Mechanisms of action of adjuvants. Front Immunol, 2013. 4: p. 114.

116. Ferreira, S.A., F.M. Gama, and M. Vilanova, Polymeric nanogels as vaccine delivery systems. Nanomedicine, 2013. 9(2): p. 159-73.

117. Morefield, G.L., et al., Role of aluminum-containing adjuvants in antigen internalization by dendritic cells in vitro. Vaccine, 2005. 23(13): p. 158895.

118. Kool, M., et al., Alum adjuvant boosts adaptive immunity by inducing uric acid and activating inflammatory dendritic cells. J Exp Med, 2008. 205(4): p. 869-82.

119. Oleszycka, E. and E.C. Lavelle, Immunomodulatory properties of the vaccine adjuvant alum. Curr Opin Immunol, 2014. 28: p. 1-5.

120. Li, H., S. Nookala, and F. Re, Aluminum hydroxide adjuvants activate caspase-1 and induce IL-1beta and IL-18 release. J Immunol, 2007. 178(8): p. 5271-6.

121. Schijns, V.E., M. Strioga, and S. Ascarateil, Oil-based emulsion vaccine adjuvants. Curr Protoc Immunol, 2014. 106: p. 218 1-7.

122. Seubert, A., et al., The adjuvants aluminum hydroxide and MF59 induce monocyte and granulocyte chemoattractants and enhance monocyte differentiation toward dendritic cells. J Immunol, 2008. 180(8): p. 540212 . 
123. Jensen, F.C., et al., Adjuvant activity of incomplete Freund's adjuvant. Adv Drug Deliv Rev, 1998. 32(3): p. 173-186.

124. Didierlaurent, A.M., et al., ASO4, an aluminum salt- and TLR4 agonistbased adjuvant system, induces a transient localized innate immune response leading to enhanced adaptive immunity. J Immunol, 2009. 183(10): p. 6186-97.

125. Morel, S., et al., Adjuvant System ASO3 containing alpha-tocopherol modulates innate immune response and leads to improved adaptive immunity. Vaccine, 2011.29(13): p. 2461-73.

126. Moser, C., et al., Influenza virosomes as vaccine adjuvant and carrier system. Expert Rev Vaccines, 2013. 12(7): p. 779-91.

127. Bungener, L., et al., Virosome-mediated delivery of protein antigens in vivo: efficient induction of class I MHC-restricted cytotoxic T lymphocyte activity. Vaccine, 2005. 23(10): p. 1232-41.

128. Bungener, L., et al., Virosome-mediated delivery of protein antigens to dendritic cells. Vaccine, 2002. 20(17-18): p. 2287-95.

129. Amigorena, S. and A. Savina, Intracellular mechanisms of antigen cross presentation in dendritic cells. Curr Opin Immunol, 2010. 22(1): p. 10917.

130. Tan, L.S., Liposomes as antigen vehicles to increase immunogenicity: effects of variation of structural characteristics. Ann Acad Med Singapore, 1991. 20(1): p. 78-83.

131. Vartak, A. and S.J. Sucheck, Recent Advances in Subunit Vaccine Carriers. Vaccines (Basel), 2016. 4(2).

132. Manolova, V., et al., Nanoparticles target distinct dendritic cell populations according to their size. Eur J Immunol, 2008. 38(5): p. 140413.

133. Christensen, D., et al., Cationic liposomes as vaccine adjuvants. Expert Rev Vaccines, 2011. 10(4): p. 513-21.

134. Hafez, I.M., N. Maurer, and P.R. Cullis, On the mechanism whereby cationic lipids promote intracellular delivery of polynucleic acids. Gene Ther, 2001. 8(15): p. 1188-96.

135. Henriksen-Lacey, M., et al., Liposomes based on dimethyldioctadecylammonium promote a depot effect and enhance immunogenicity of soluble antigen. J Control Release, 2010. 142(2): p. 180-6.

136. Levy, D.A., et al., Phase II study of D.651, an oral vaccine designed to prevent recurrences of vulvovaginal candidiasis. Vaccine, 1989. 7(4): p. 337-40.

137. De Bernardis, F., et al., A virosomal vaccine against candidal vaginitis: immunogenicity, efficacy and safety profile in animal models. Vaccine, 2012. 30(30): p. 4490-8.

138. De Bernardis, F., et al., Studies of Immune Responses in Candida vaginitis. Pathogens, 2015.4(4): p. 697-707. 
139. Cutler, J.E., et al., Horizontal transmission of Candida albicans and evidence of a vaccine response in mice colonized with the fungus. PLoS One, 2011. 6(7): p. e22030.

140. Lamas, B., et al., CARD9 impacts colitis by altering gut microbiota metabolism of tryptophan into aryl hydrocarbon receptor ligands. Nat Med, 2016.

141. Richard, M.L., et al., Gut fungal microbiota: the Yin and Yang of inflammatory bowel disease. Inflamm Bowel Dis, 2015. 21(3): p. 656-65.

142. Jostins, L., et al., Host-microbe interactions have shaped the genetic architecture of inflammatory bowel disease. Nature, 2012. 491(7422): p. 119-24.

143. Quinton, J.F., et al., Anti-Saccharomyces cerevisiae mannan antibodies combined with antineutrophil cytoplasmic autoantibodies in inflammatory bowel disease: prevalence and diagnostic role. Gut, 1998. 42(6): p. 788-91.

144. Pericolini, E., et al., Secretory Aspartyl Proteinases Cause Vaginitis and Can Mediate Vaginitis Caused by Candida albicans in Mice. MBio, 2015. 6(3): p. e00724.

145. Stawerska, R., et al., Prevalence of autoantibodies against some selected growth and appetite-regulating neuropeptides in serum of short children exposed to Candida albicans colonization and/or Helicobacter pylori infection: the molecular mimicry phenomenon. Neuro Endocrinol Lett, 2015. 36(5): p. 458-64. 